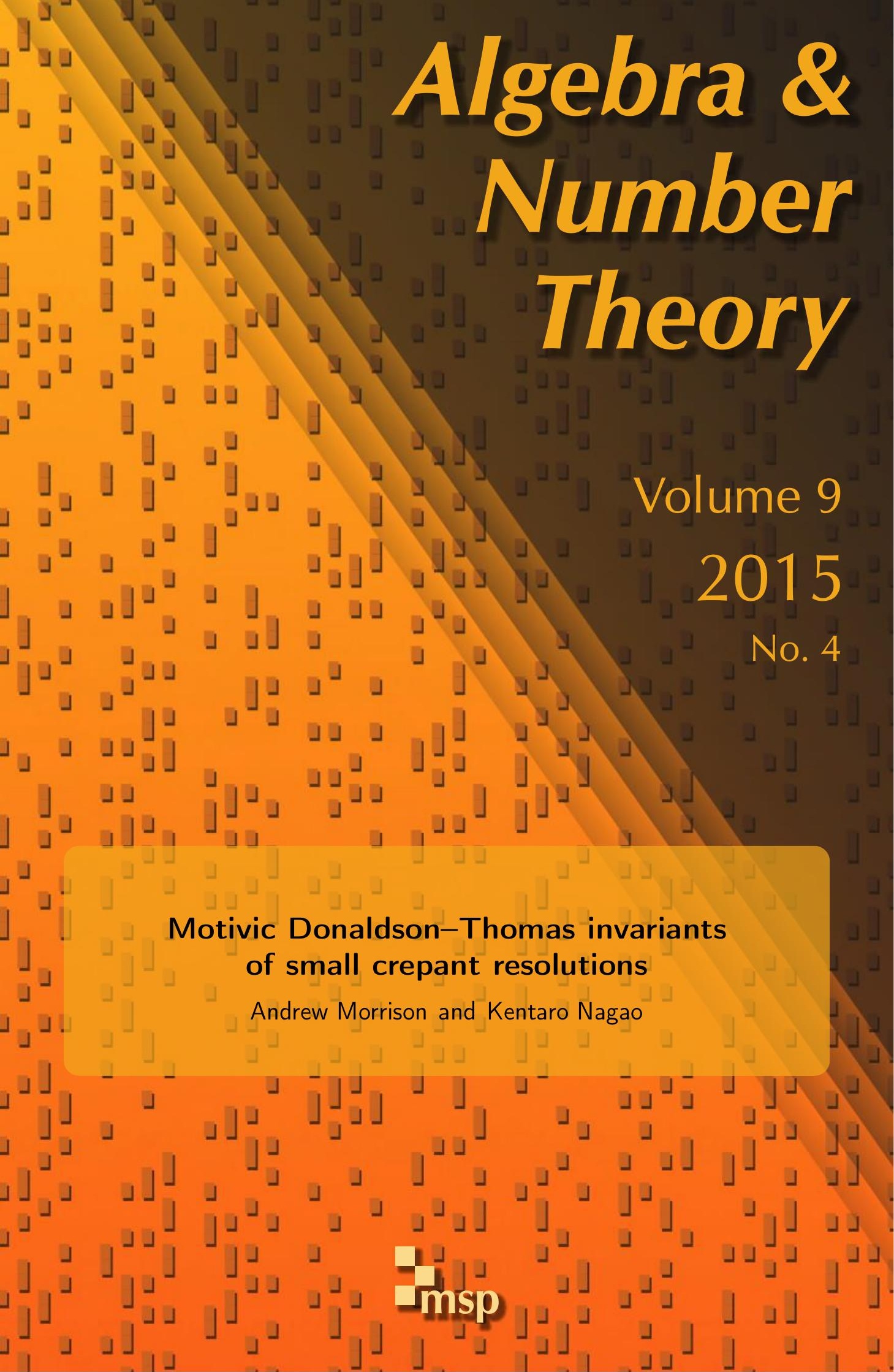




\title{
Motivic Donaldson-Thomas invariants of small crepant resolutions
}

\author{
Andrew Morrison and Kentaro Nagao
}

We compute the motivic Donaldson-Thomas theory of a small crepant resolution of a toric Calabi-Yau 3-fold.

Introduction 767

Main result 769

Corollaries

1. Root system of type $\tilde{A}_{N}$

2. Noncommutative crepant resolutions

3. Motivic Donaldson-Thomas invariants

4. The universal DT series: special case

5. The universal DT series: general case

6. Motivic DT with framing and DT/PT series

Appendix

Acknowledgements

Biographical note

References
770

773

773

779

782

797

798

800

810

811

811

\section{Introduction}

This paper is a continuation of [Morrison et al. 2012]. We study the motivic Donaldson-Thomas invariants of noncommutative and commutative crepant resolutions of the affine toric Calabi-Yau 3-fold $\left\{X Y-Z^{N_{0}} W^{N_{1}}\right\} \subset \mathbb{C}^{4}$.

A Donaldson-Thomas (DT) invariant of a Calabi-Yau 3-fold $Y$ is a counting invariant of coherent sheaves on $Y$, introduced in [Thomas 2000] as a holomorphic analogue of the Casson invariant of a real 3-manifold. A component of the moduli space of stable coherent sheaves on $Y$ carries a symmetric obstruction theory and a virtual fundamental cycle [Behrend and Fantechi 1997; 2008]. A DT invariant of a compact $Y$ is then defined as the integral of the constant function 1 over the virtual fundamental cycle of the moduli space.

MSC2010: 14N35.

Keywords: motivic Donaldson-Thomas invariants, small crepant resolutions. 

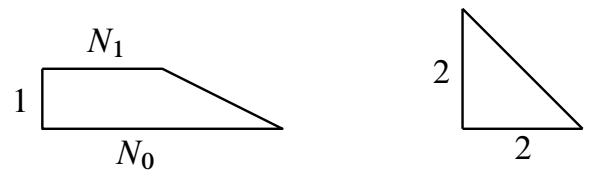

Figure 1. Polygons for $\mathcal{X}_{N_{0}, N_{1}}$ and $\mathcal{X}_{(\mathbb{Z} / 2 \mathbb{Z})^{2}}$.

It is known that the moduli space of coherent sheaves on $Y$ can be locally described as the critical locus of a function, the holomorphic Chern-Simons (CS) functional (see [Joyce and Song 2012]). Behrend [2009] provided a description of DT invariants in terms of the Euler characteristic of the Milnor fiber of the CS functional. Inspired by this result, the proposal of [Kontsevich and Soibelman 2008; Behrend et al. 2013] was to study the motivic Milnor fiber of the CS functional as a motivic refinement of the DT invariant. Such a refinement had been expected in string theory [Iqbal et al. 2009; Dimofte and Gukov 2010].

On the other hand, in [Szendrói 2008] it was proposed to study counting invariants for the noncommutative crepant resolution (NCCR) of the conifold, which are called noncommutative Donaldson-Thomas (NCDT) invariants. It was also conjectured there that NCDT and DT invariants are related by wall-crossing. The paper [Nagao and Nakajima 2011] realized this, by:

- Describing the chamber structure on the space of stability parameters for the NCCR.

- Finding chambers which correspond to geometric DT and stable pair (PT) invariants, as well as NCDT invariants.

- Computing the generating function of DT-type invariants for each chamber.

For the conifold, the dimension of the fiber of the crepant resolution is less than 2 (we say that the resolution is small). This condition plays an important role in many places of the paper. Affine toric Calabi-Yau 3-folds which have small crepant resolutions are classified as follows:

(1) $\mathcal{X}=\mathcal{X}_{N_{0}, N_{1}}:=\left\{X Y-Z^{N_{0}} W^{N_{1}}\right\}$ for $N_{0}>0$ and $N_{1} \geq 0$.

(2) $\mathcal{X}=\mathcal{X}_{(\mathbb{Z} / 2 \mathbb{Z})^{2}}:=\mathbb{C}^{3} /(\mathbb{Z} / 2 \mathbb{Z})^{2}$, where $(\mathbb{Z} / 2 \mathbb{Z})^{2}$ acts on $\mathbb{C}^{3}$ with weights $(1,0)$, $(0,1)$ and $(1,1)$.

In [Nagao 2012], counting invariants for noncommutative and commutative crepant resolutions of $\left\{X Y-Z^{N_{0}} W^{N_{1}}\right\}$ were studied. First, we provided descriptions of NCCRs of $\left\{X Y-Z^{N_{0}} W^{N_{1}}\right\}$ in terms of a quiver with potential. Given $N_{0}$ and $N_{1}$, the quivers with potential are not unique. However it was also shown that any such quivers with potential are related by a sequence of mutations. Finally, generalizations of the results in [Nagao and Nakajima 2011] are given. 
In [Morrison et al. 2012], we provided motivic refinements of formulae in [Nagao and Nakajima 2011]. For the proof, we needed one explicit evaluation of the "universal" series [Morrison et al. 2012, §2] and a wall-crossing argument [Morrison et al. 2012, §3].

In this paper, we will show similar formulae for $\left\{X Y-Z^{N_{0}} W^{N_{1}}\right\}$, that is, motivic refinements of the formulae in [Nagao 2012]. The wall-crossing argument works without modifications (Section 6), while the evaluation part is more involved (Theorem 0.1). Our strategy is as follows:

- First, in Section 4, we evaluate the universal series for a specific NCCR using a generalization of the calculation of [Morrison et al. 2012, §2.2].

- Then, in Section 5, we evaluate the universal series for a general NCCR. Nagao [2011c] provided a formula which describes how the universal series changes under mutation. Although it is assumed that the quiver has no loops and 2cycles in [Nagao 2011c], we can apply a parallel argument in our setting as well.

Since any two NCCRs are related by a sequence of mutations, the evaluation is done.

\section{Main result}

Let $\Gamma$ be a quadrilateral (or a triangle in the case $N_{1}=0$ ) as in Figure 1 and $\sigma$ a partition of $\Gamma$, that is, a division of $\Gamma$ into an $N$-tuple of triangles with area $1 / 2$, as in Figure 2. We will associate $\sigma$ to a quiver with superpotential $\left(Q_{\sigma}, \omega_{\sigma}\right)$ (see Section $2 \mathrm{~A}$ ). The set of vertices of the quiver $Q_{\sigma}$ is $\hat{I}:=\mathbb{Z} / N \mathbb{Z}$, which is identified with $\{0, \ldots, N-1\}$. A vertex has a loop if and only if it is in the subset $\hat{I}_{r} \subset \hat{I}$ (see (2-1) for the definition). It is shown in [Nagao 2012, §1] that the Jacobian algebra $J_{\sigma}:=J\left(Q_{\sigma}, \omega_{\sigma}\right)$ is an NCCR of

$$
\mathcal{X}:=\operatorname{Spec}\left(\mathbb{C}[X, Y, Z, W] /\left(X Y-Z^{N_{0}} W^{N_{1}}\right)\right) .
$$

Let $\Delta$ be the set of roots of type $\widetilde{A}_{N}$ and let $\Delta_{\sigma,+} \Delta_{\sigma,+}^{\mathrm{re}}$, and $\Delta_{\sigma,+}^{\mathrm{im}}$ denote the set of positive, positive real, and positive imaginary roots, respectively. ${ }^{1}$

For $\alpha \in \mathbb{N}^{\widehat{I}}$, let $\mathfrak{M}\left(J_{\sigma}, \alpha\right)$ be the moduli stack of $J_{\sigma}$-modules $V$ with $\underline{\operatorname{dim}} V=\alpha$. We define the generating series of the motivic DT invariants of $\left(Q_{\sigma}, W_{\sigma}\right)$ by

$$
A_{U}^{\sigma}(y)=A_{U}^{\sigma}\left(y_{0}, \ldots, y_{N-1}\right):=\sum_{\alpha \in \mathbb{N} Q_{0}}\left[\mathfrak{M}\left(J_{\sigma}, \alpha\right)\right]_{\mathrm{vir}} \cdot y^{\alpha} \in \mathcal{M}_{\mathbb{C}} \llbracket y_{0}, \ldots, y_{N-1} \rrbracket \cdot{ }^{2}
$$

\footnotetext{
${ }^{1}$ From the view point of the root system, a choice of a partition $\sigma$ corresponds to a choice of a set of simple roots.

${ }^{2}$ For the wall-crossing of motivic DT theory, a twisted product on the $y_{\alpha}$ twisted by the Euler form plays a crucial role. In this case, the twisted product coincides with the usual commutative product since the Euler form is trivial.
} 
Here $y^{\alpha}:=\prod\left(y_{i}\right)^{\alpha_{i}}$ and $[\bullet]_{\text {vir }}$ denotes the virtual motive (see Section 3A), an element of a suitable ring of motives $\mathcal{M}_{\mathbb{C}}$. The subscript refers to the fact that we think of this series as the universal series.

To each root $\alpha \in \Delta_{\sigma,+}$ we associate an infinite product as follows:

- For a real root $\alpha \in \Delta_{\sigma,+}^{\mathrm{re}}$ such that $\sum_{k \notin \widehat{I}_{r}} \alpha_{k}$ is odd, put

$$
A^{\alpha}(y):=\operatorname{Exp}\left(\frac{-\mathbb{L}^{-\frac{1}{2}}}{1-\mathbb{L}^{-1}} y^{\alpha}\right)=\prod_{j \geq 0}\left(1-\mathbb{L}^{-j-\frac{1}{2}} y^{\alpha}\right) .
$$

- For a real root $\alpha \in \Delta_{\sigma,+}^{\mathrm{re}}$ such that $\sum_{k \notin \widehat{I}_{r}} \alpha_{k}$ is even, put

$$
A^{\alpha}(y):=\operatorname{Exp}\left(\frac{1}{1-\mathbb{L}^{-1}} y^{\alpha}\right)=\prod_{j \geq 0}\left(1-\mathbb{L}^{-j} y^{\alpha}\right)^{-1} .
$$

- For an imaginary root $\alpha \in \Delta_{\sigma,+}^{\mathrm{im}}$, put

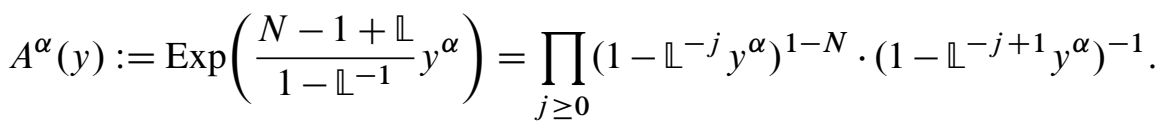

The main result of this paper is the following formula:

\section{Theorem 0.1.}

$$
A_{U}^{\sigma}(y)=\prod_{\alpha \in \Delta_{\sigma,+}} A^{\alpha}(y) .
$$

This is proved in Section 4 and Section 5B.

\section{Corollaries}

Let $\widetilde{J}_{\sigma}=J\left(\widetilde{Q}_{\sigma}, W_{\sigma}\right)$ be the framed algebra given by adding the new vertex $\infty$ and the new arrow from $\infty$ to 0 to the quiver of $J_{\sigma}$. Nagao and Nakajima [2011] introduced a notion of $\zeta$-(semi)stability of $\widetilde{J}$-modules $\widetilde{V}$ with $\operatorname{dim} \widetilde{V}_{\infty} \leq 1$ for a stability parameter $\zeta \in \mathbb{R}^{\hat{I}}$.

For $\alpha \in \mathbb{N}^{\widehat{I}}$, let $\mathfrak{M}_{\zeta}(\widetilde{J}, \alpha)$ be the moduli space of $\zeta$-stable $\widetilde{J}$-modules $\tilde{V}$ with $\underline{\operatorname{dim}} \tilde{V}=(\alpha, 1)$. We want to compute the motivic generating series

$$
Z_{\zeta}(y)=Z_{\zeta}\left(y_{0}, \ldots, y_{N-1}\right):=\sum_{\alpha \in \mathbb{N} T}\left[\mathfrak{M}_{\zeta}(\widetilde{J}, \alpha)\right]_{\mathrm{vir}} \cdot y^{\alpha} \in \mathcal{M}_{\mathbb{C}} \llbracket y_{0}, \ldots, y_{N-1} \rrbracket .
$$

For each root $\alpha \in \Delta_{\sigma,+}$, we put

$$
Z_{\alpha}\left(y_{0}, \ldots, y_{N-1}\right):=\frac{A^{\alpha}\left(-\mathbb{L}^{\frac{1}{2}} y_{0}, y_{1}, \ldots, y_{N-1}\right)}{A^{\alpha}\left(-\mathbb{L}^{-\frac{1}{2}} y_{0}, y_{1}, \ldots, y_{N-1}\right)} .
$$

These are given as follows: 
- For a real root $\alpha \in \Delta_{\sigma,+}^{\mathrm{re}}$ such that $\sum_{k \notin \widehat{I}_{r}} \alpha_{k}$ is odd, we have

$$
Z_{\alpha}\left(-y_{0}, \ldots, y_{N-1}\right)=\prod_{i=0}^{\alpha_{0}-1}\left(1-\mathbb{Q}^{-\frac{\alpha_{0}}{2}+\frac{1}{2}+i} y^{\alpha}\right) .
$$

- For a real root $\alpha \in \Delta_{\sigma,+}^{\mathrm{re}}$ such that $\sum_{k \notin \widehat{I}_{r}} \alpha_{k}$ is even, we have

$$
Z_{\alpha}\left(-y_{0}, \ldots, y_{N-1}\right)=\prod_{i=0}^{\alpha_{0}-1}\left(1-\mathbb{L}^{-\frac{\alpha_{0}}{2}+1+i} y^{\alpha}\right)^{-1} .
$$

- For an imaginary root $\alpha \in \Delta_{\sigma,+}^{\mathrm{im}}$, we have

$$
Z_{\alpha}\left(-y_{0}, \ldots, y_{N-1}\right)=\prod_{i=0}^{\alpha_{0}-1}\left(1-\mathbb{1}^{-\frac{\alpha_{0}}{2}+1+i} y^{\alpha}\right)^{1-N} \cdot\left(1-\mathbb{1}^{-\frac{\alpha_{0}}{2}+2+j} y^{\alpha}\right)^{-1} .
$$

Applying the same argument as [Morrison et al. 2012, §3], we get the following formula (Section 6A):

Corollary 0.2. For $\zeta \in \mathbb{R}^{\widetilde{I}}$ not orthogonal to any root, we have

$$
Z_{\zeta}(y)=\prod_{\substack{\alpha \in \Delta_{\sigma,+} \\ \zeta \cdot \alpha<0}} Z_{\alpha}\left(y_{0}, \ldots, y_{N-1}\right) .
$$

By [Behrend 2009; Behrend et al. 2013], the specialization $\left.Z_{\zeta}(y)\right|_{\mathbb{L}^{\frac{1}{2}} \rightarrow 1}$ is the DT-type series at the generic stability parameter $\zeta$, computed in [Nagao 2012].

Let $\mathcal{Y}_{\sigma} \rightarrow \mathcal{X}$ be the crepant resolution corresponding to $\sigma$. The noncommutative crepant resolution $J_{\sigma}$ is derived equivalent to $\mathcal{Y}_{\sigma}$. In [Nagao and Nakajima 2011, $\S 3]$, we found a stability parameter $\zeta_{\mathrm{DT}}$ (resp. $\zeta_{\mathrm{PT}}$ ) such that the moduli space coincides with the Hilbert scheme (resp. the stable pair moduli space) for $\mathcal{Y}_{\sigma}$.

Let $Z_{\mathrm{DT}}^{\sigma}\left(s, T_{1}, \ldots, T_{N-1}\right)$ and $Z_{\mathrm{DT}}^{\sigma}\left(s, T_{1}, \ldots, T_{N-1}\right)$ be the generating functions of DT and PT invariants of $\mathcal{Y}_{\sigma}$, respectively. Here $s$ is the variable for the homology class of a point and $T_{i}$ is the variable for the homology class of the $i$-th component $C_{i}$ of the exceptional curve. The variable change induced by the derived equivalence is given by

$$
s:=y_{0} \cdot y_{1} \cdots y_{N-1}, \quad T_{i}=y_{i} .
$$

For $1 \leq a \leq b \leq N-1$, we put

$$
C_{[a, b]}:=\left[C_{a}\right]+\cdots+\left[C_{b}\right] \in H_{2}\left(\mathcal{Y}_{\sigma}, \mathbb{Z}\right),
$$

where $C_{i}$ is a component of the exceptional curve, and let

$$
T_{[a, b]}=T_{a} \cdots T_{b}
$$


be the corresponding monomial. Let $c(a, b)$ denote the number of $(-1,-1)$-curves in $\left\{C_{i} \mid a \leq i \leq b\right\}$. We define infinite products as follows:

- If $c(a, b)$ is odd, we put

$$
Z_{[a, b]}=Z_{[a, b]}\left(s, T_{[a, b]}\right):=\prod_{n=1}^{\infty}\left(\prod_{i=0}^{n-1}\left(1-\mathbb{L}^{-\frac{n}{2}+\frac{1}{2}+i} \cdot(-s)^{n} \cdot T_{[a, b]}\right)\right) .
$$

- If $c(a, b)$ is even, we put

$$
Z_{[a, b]}=Z_{[a, b]}\left(s, T_{[a, b]}\right):=\prod_{n=1}^{\infty}\left(\prod_{i=0}^{n-1}\left(1-\mathbb{L}^{-\frac{n}{2}+1+i} \cdot(-s)^{n} \cdot T_{[a, b]}\right)^{-1}\right) .
$$

- For imaginary roots, we put

$Z_{\mathrm{im}}=Z_{\mathrm{im}}(s):=\prod_{n=1}^{\infty}\left(\prod_{i=0}^{n-1}\left(1-\mathbb{\mathbb { L }}^{-\frac{n}{2}+1+i}(-s)^{n}\right)^{1-N}\left(1-\mathbb{1}^{-\frac{n}{2}+2+i}(-s)^{n}\right)^{-1}\right)$.

Corollary 0.3. (1) The refined $D T$ and $P T$ series of $\mathcal{Y}_{\sigma}$ are given by the formulae

$$
\begin{aligned}
& Z_{\mathrm{DT}}\left(s, T_{1}, \ldots, T_{N-1}\right)=Z_{\mathrm{im}}(s) \cdot \prod_{1 \leq a \leq b \leq N-1} Z_{[a, b]}\left(s, T_{[a, b]}\right), \\
& Z_{\mathrm{PT}}\left(s, T_{1}, \ldots, T_{N-1}\right)=\prod_{1 \leq a \leq b \leq N-1} Z_{[a, b]}\left(s, T_{[a, b]}\right) .
\end{aligned}
$$

(2) The generating function of virtual motives of the Hilbert scheme of points on $\mathcal{Y}_{\sigma}$ is given by the formula

$$
Z_{0-\operatorname{dim}}(s):=\sum_{n=0}^{\infty}\left[\left(\mathcal{Y}_{\sigma}\right)^{[n]}\right]_{\mathrm{vir}} \cdot s^{n}=Z_{\mathrm{im}}
$$

(3) The refined version of the DT-PT correspondence for $\mathcal{Y}_{\sigma}$ holds:

$$
Z_{\mathrm{DT}}\left(s, T_{1}, \ldots, T_{N-1}\right)=Z_{0-\operatorname{dim}}(s) \times Z_{\mathrm{PT}}\left(s, T_{1}, \ldots, T_{N-1}\right) .
$$

Remark. The formula in (2) is a direct consequence of the formula for $Z_{\mathrm{DT}}$ in (1), since the polynomial in the $T_{[a, b]}$ variables does not contribute. 


\section{Root system of type $\tilde{A}_{N}$}

Let $N_{0}>0$ and $N_{1} \geq 0$ be integers such that $N_{0} \geq N_{1}$, and set $N=N_{0}+N_{1}$. We set

$$
\begin{aligned}
& I=\{1, \ldots, N-1\}, \\
& \widehat{I}=\{0,1, \ldots, N-1\}, \\
& \widetilde{I}=\left\{\frac{1}{2}, \frac{3}{2}, \ldots, N-\frac{1}{2}\right\}, \\
& \widetilde{\mathbb{Z}}=\left\{n+\frac{1}{2} \mid n \in \mathbb{Z}\right\} .
\end{aligned}
$$

For $l \in \mathbb{Z}$ and $j \in \widetilde{\mathbb{Z}}$, let $\underline{l} \in \widehat{I}$ and $\underline{j} \in \tilde{I}$ be the elements such that $l-\underline{l} \equiv j-\underline{j} \equiv 0$ modulo $N$.

Let $\mathbb{Z}^{\hat{I}}$ be the free abelian group with basis $\left\{\alpha_{i} \mid i \in \widehat{I}\right\}$; we call $\alpha_{i}$ a simple root. We put

$$
\begin{aligned}
\Delta_{+}^{\mathrm{fin}} & :=\left\{\alpha_{[a, b]}:=\alpha_{a}+\cdots+\alpha_{b} \mid 1 \leq a \leq b \leq N-1\right\}, \\
\Delta_{+}^{\mathrm{re},+} & :=\left\{\alpha_{[a, b]}+n \cdot \delta \mid \alpha_{[a, b]} \in \Delta_{+}^{\mathrm{fin}}, n \in \mathbb{Z}_{\geq 0}\right\}, \\
\Delta_{+}^{\mathrm{re},-} & :=\left\{-\alpha_{[a, b]}+n \cdot \delta \mid \alpha_{[a, b]} \in \Delta_{+}^{\mathrm{fin}}, n \in \mathbb{Z}_{>0}\right\},
\end{aligned}
$$

and

$$
\Delta_{+}^{\mathrm{re}}:=\Delta_{+}^{\mathrm{re},+} \sqcup \Delta_{+}^{\mathrm{re},-}, \quad \Delta_{+}^{\mathrm{im}}:=\left\{n \cdot \delta \mid n \in \mathbb{Z}_{>0}\right\},
$$

where $\delta:=\alpha_{0}+\cdots+\alpha_{N-1}$ is the (positive minimal) imaginary root.

For $k \in \hat{I}$, the simple reflection at $k$ is the group homomorphism given by

$$
\begin{aligned}
\mathbb{Z}^{\hat{I}} & \rightarrow \mathbb{Z}^{\hat{I}}, \\
\alpha_{i} & \mapsto \alpha_{i}-C_{i k} \cdot \alpha_{k},
\end{aligned}
$$

where $C$ is the Cartan matrix of type $\tilde{A}_{N}$. This gives a self-bijection of $\Delta_{+}^{\mathrm{re},+} \backslash\left\{\alpha_{k}\right\}$.

\section{Noncommutative crepant resolutions}

2A. Quivers with potential. We denote by $\Gamma$ the quadrilateral (or the triangle in case $\left.N_{1}=0\right)$ with vertices $(0,0),(0,1),\left(N_{0}, 0\right)$ and $\left(N_{1}, 1\right)$. Note that the affine toric Calabi-Yau 3-fold corresponding to $\Gamma$ is $\mathcal{X}=\left\{X Y-Z^{N_{0}} W^{N_{1}}\right\}$.

A partition $\sigma$ of $\Gamma$ is a pair of functions $\sigma_{x}: \widetilde{I} \rightarrow \widetilde{\mathbb{Z}}$ and $\sigma_{y}: \widetilde{I} \rightarrow\{0,1\}$ such that:

- $\sigma(i):=\left(\sigma_{x}(i), \sigma_{y}(i)\right)$ gives a bijection between $\tilde{I}$ and

$$
\left\{\left(\frac{1}{2}, 0\right),\left(\frac{3}{2}, 0\right), \ldots,\left(N_{0}-\frac{1}{2}, 0\right),\left(\frac{1}{2}, 1\right),\left(\frac{3}{2}, 1\right), \ldots,\left(N_{1}-\frac{1}{2}, 1\right)\right\} .
$$

- If $i<j$ and $\sigma_{y}(i)=\sigma_{y}(j)$ then $\sigma_{x}(i)>\sigma_{x}(j)$. 


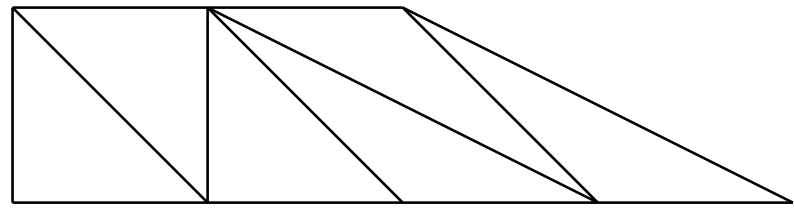

Figure 2. $\Gamma_{\sigma}$.

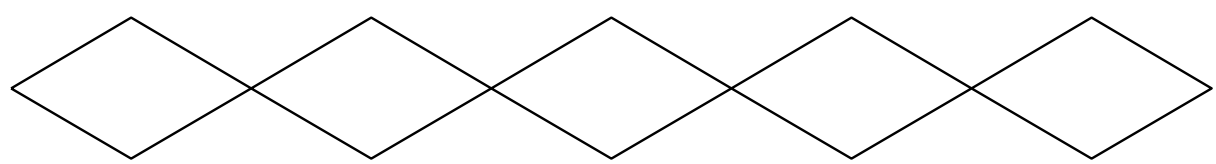

Figure 3. $S$.

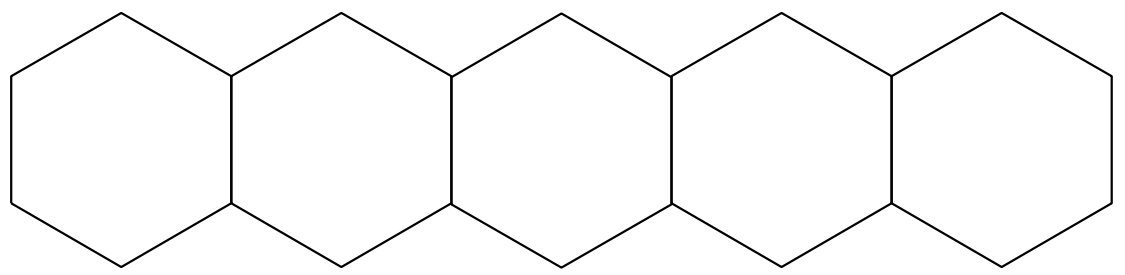

Figure 4. $H$.

Giving a partition $\sigma$ of $\Gamma$ is equivalent to dividing $\Gamma$ into an $N$-tuple of triangles $\left\{T_{i}\right\}_{i \in \tilde{I}}$ with area $1 / 2$ so that $T_{i}$ has $\left(\sigma_{x}(i) \pm 1 / 2, \sigma_{y}(i)\right)$ as its vertices. Let $\Gamma_{\sigma}$ be the corresponding diagram, $\Delta_{\sigma}$ the fan and $f_{\sigma}: \mathcal{Y}_{\sigma} \rightarrow \mathcal{X}$ the crepant resolution of $\mathcal{X}$. We put

$$
\widehat{I}_{r}:=\left\{k \in \widehat{I} \mid \sigma_{y}\left(k-\frac{1}{2}\right)=\sigma_{y}\left(k+\frac{1}{2}\right)\right\} .
$$

Example 1. Let us consider as an example the case $N_{0}=4, N_{1}=2$ and

$$
(\sigma(i))_{i \in \tilde{I}}=\left(\left(\frac{7}{2}, 0\right),\left(\frac{3}{2}, 1\right),\left(\frac{5}{2}, 0\right),\left(\frac{3}{2}, 0\right),\left(\frac{1}{2}, 1\right),\left(\frac{1}{2}, 0\right)\right) .
$$

We show the corresponding diagram $\Gamma_{\sigma}$ in Figure 2.

Let $S$ be the union of an infinite number of rhombi with edge length 1, as in Figure 3, located so that the centers of the rhombi are on a line parallel to the $x$-axis in $\mathbb{R}^{2}$, and let $H$ be the union of an infinite number of hexagons with edge length 1 , as in Figure 4 located so that the centers of the hexagons are in a line parallel to the $x$-axis in $\mathbb{R}^{2}$.

We form the sequence $\tau=\tau_{\sigma}: \mathbb{Z} \rightarrow\{S, H\}$ which maps $l$ to $S$ (resp. $H$ ) if $l$ modulo $N$ is not in $\hat{I}_{r}$ (resp. is in $\widehat{I}_{r}$ ), and cover the whole plane $\mathbb{R}^{2}$ by arranging $S$ 's and $H$ 's according to this sequence (see Figure 5). We regard this as a graph 


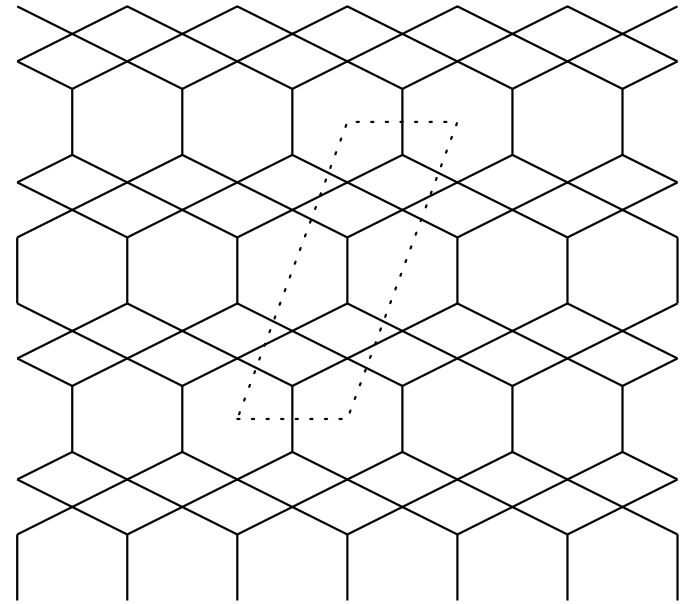

Figure 5. $P_{\sigma}$ in the case of Example 1.

on the 2-dimensional torus $\mathbb{R}^{2} / \Lambda$, where $\Lambda$ is the lattice generated by $(\sqrt{3}, 0)$ and $\left(N_{0}-N_{1},\left(N_{0}-N_{1}\right) \sqrt{3}+N_{1}\right)$.

We can color the vertices of this graph black or white so that each edge connects a black vertex and a white one. Let $P_{\sigma}$ denote this bipartite graph on the torus. For each edge $h^{\vee}$ in $P_{\sigma}$, we make its dual edge $h$ directed so that we see the black end of $h^{\vee}$ on our right-hand side when we cross $h^{\vee}$ along $h$ in the given direction. Let $Q_{\sigma}$ denote the resulting quiver. The set of vertices of the quiver $Q_{\sigma}$ is $\hat{I}$, which is identified with $\mathbb{Z} / N \mathbb{Z}$. The set of edges of the quiver $Q_{\sigma}$ is given by

$$
H:=\left(\coprod_{i \in \widetilde{I}} h_{i}^{+}\right) \sqcup\left(\coprod_{i \in \widetilde{I}} h_{i}^{-}\right) \sqcup\left(\coprod_{k \in \widehat{I}_{r}} r_{k}\right) .
$$

Here $h_{i}^{+}$(resp. $h_{i}^{-}$) is an edge from $i-\frac{1}{2}$ to $i+\frac{1}{2}$ (resp. from $i+\frac{1}{2}$ to $i-\frac{1}{2}$ ), and $r_{k}$ is an edge from $k$ to itself.

For each vertex $q$ of $P_{\sigma}$, let $\omega_{q}$ be the potential ${ }^{3}$ which is the composition of all arrows in $Q_{\sigma}$ corresponding to edges in $P_{\sigma}$ with $q$ as their ends. We define

$$
\omega_{\sigma}:=\sum_{q \text { black }} \omega_{q}-\sum_{q \text { white }} \omega_{q} .
$$

The relations of the Jacobian algebra are as follows:

${ }^{3}$ A potential of a quiver $Q$ is an element in $\mathbb{C} Q /[\mathbb{C} Q, \mathbb{C} Q]$, i.e., a linear combination of equivalence classes of cyclic paths in $Q$, where two paths are equivalent if they coincide after a cyclic rotation. 
- For $i \in \tilde{I}$ such that $i-\frac{1}{2}, i+\frac{1}{2} \in \widehat{I}_{r}$,

$$
h_{i}^{+} \circ r_{i-\frac{1}{2}}=r_{i+\frac{1}{2}} \circ h_{i}^{+} \text {and } r_{i-\frac{1}{2}} \circ h_{i}^{-}=h_{i}^{-} \circ r_{i+\frac{1}{2}} \text {. }
$$

- For $i \in \tilde{I}$ such that $i-\frac{1}{2} \in \widehat{I}_{r}$ and $i+\frac{1}{2} \notin \hat{I}_{r}$,

$$
h_{i}^{+} \circ r_{i-\frac{1}{2}}=h_{i+1}^{-} \circ h_{i+1}^{+} \circ h_{i}^{+} \text {and } r_{i-\frac{1}{2}} \circ h_{i}^{-}=h_{i}^{-} \circ h_{i+1}^{-} \circ h_{i+1}^{+} \text {. }
$$

- For $i \in \tilde{I}$ such that $i-\frac{1}{2} \notin \widehat{I}_{r}$ and $i+\frac{1}{2} \in \hat{I}_{r}$,

$$
h_{i}^{+} \circ h_{i-1}^{+} \circ h_{i-1}^{-}=r_{i+\frac{1}{2}} \circ h_{i}^{+} \quad \text { and } h_{i-1}^{+} \circ h_{i-1}^{-} \circ h_{i}^{-}=h_{i}^{-} \circ r_{i+\frac{1}{2}} \text {. }
$$

- For $i \in \tilde{I}$ such that $i-\frac{1}{2}, i+\frac{1}{2} \notin \widehat{I}_{r}$,

$h_{i}^{+} \circ h_{i-1}^{+} \circ h_{i-1}^{-}=h_{i+1}^{-} \circ h_{i+1}^{+} \circ h_{i}^{+}$and $h_{i-1}^{+} \circ h_{i-1}^{-} \circ h_{i}^{-}=h_{i}^{-} \circ h_{i+1}^{-} \circ h_{i+1}^{+}$.

- For $k \in \hat{I}_{r}$,

$$
h_{i-\frac{1}{2}}^{+} \circ h_{i-\frac{1}{2}}^{-}=h_{i+\frac{1}{2}}^{-} \circ h_{i+\frac{1}{2}}^{+} .
$$

2B. NCCR and derived equivalence. Let $\pi: \mathcal{Y}_{\sigma} \rightarrow \mathcal{X}$ be the crepant resolution corresponding to $\sigma$.

Theorem 2.1 [Nagao 2012, Theorems 1.15 and 1.20].

$$
D^{b}\left(\bmod J_{\sigma}\right) \simeq D^{b}\left(\operatorname{Coh} \mathcal{Y}_{\sigma}\right)
$$

The equivalence is given by an explicit tilting vector bundle which is a direct sum of line bundles [Nagao 2012, Theorem 1.10]. In particular, the following map is compatible with the derived equivalence

$$
\begin{aligned}
H^{0}\left(Y_{\sigma}, \mathbb{Z}\right) \oplus H^{2}\left(Y_{\sigma}, \mathbb{Z}\right) & \rightarrow \mathbb{Z}^{I}, \\
{[\mathrm{pt}] } & \mapsto \delta, \\
{\left[C_{i}\right] } & \mapsto \alpha_{i},
\end{aligned}
$$

where $\alpha_{i}$ is the $i$-th fundamental vector and $\delta:=\alpha_{0}+\alpha_{1}+\cdots+\alpha_{N-1}$.

2C. Mutation and derived equivalence. The Derksen-Weyman-Zelevinsky mutation [Derksen et al. 2010] of a quiver with a potential induces a derived equivalence of the derived categories of Ginzburg's differential graded algebras [Keller and Yang 2011]. Moreover, the relation between the module categories of Jacobian algebras has a description in terms of torsion pair and tilting, which plays a crucial role for the wall-crossing formulae [Kontsevich and Soibelman 2008; Nagao 2013]. In this paper, we cannot apply [Derksen et al. 2010; Keller and Yang 2011] since we have loops and oriented 2-cycles in the quiver. In this subsection, we see derived 
equivalences and descriptions of module categories using the explicit computations given in [Nagao 2012, §3].

Let $k$ be an edge of the partition $\sigma$ which is a diagonal of a parallelogram. Note that such a $k$ corresponds to a vertex without loops. Let $\sigma^{\prime}$ denote the partition which is obtained by a "flip" of the edge $k$.

Let $P_{i}$ be the indecomposable projective $J_{\sigma}$-module associated to a vertex $i$. Note that, as a vector space, $P_{i}$ is the space of linear combinations of paths ending at the vertex $i$. We define

$$
P_{k}^{\prime}:=\operatorname{coker}\left(P_{k} \rightarrow P_{k-1} \oplus P_{k+1}\right),
$$

and put $P_{i}^{\prime}=P_{i}$ for $i \neq k$. Here the map $P_{k} \rightarrow P_{k \pm 1}$ above is induced by the arrow from $k$ to $k \pm 1$.

Theorem 2.2 [Nagao 2012, Proposition 3.1].

$$
\operatorname{End}\left(\oplus P_{i}^{\prime}\right)^{\mathrm{op}} \simeq J_{\sigma^{\prime}}
$$

(2) The map

$$
\Phi_{k}:=\mathbf{R} \operatorname{Hom}\left(\oplus P_{i}^{\prime}, \bullet\right): D^{b}\left(\bmod J_{\sigma}\right) \rightarrow D^{b}\left(\bmod J_{\sigma^{\prime}}\right)
$$

is an equivalence.

For a $J_{\sigma}$-module $V=\bigoplus_{i \in \hat{I}} V_{i}$, we have

$$
\left(H_{\bmod J_{\sigma^{\prime}}}^{j}\left(\Phi_{k}(V)\right)\right)_{i}= \begin{cases}V_{i} & i \neq k, j=0, \\ \operatorname{ker}\left(V_{k-1} \oplus V_{k+1} \rightarrow V_{k}\right) & i=k, j=0, \\ \operatorname{coker}\left(V_{k-1} \oplus V_{k+1} \rightarrow V_{k}\right) & i=k, j=1, \\ 0 & \text { otherwise }\end{cases}
$$

The simple reflection is compatible with the derived equivalence for dimension vectors.

By the description above, we have

$$
\begin{aligned}
\bmod J_{\sigma} \cap \Phi_{k}^{-1}\left(\bmod J_{\sigma^{\prime}}\right) & =\left\{V \in \bmod J_{\sigma} \mid \operatorname{coker}\left(V_{k-1} \oplus V_{k+1} \rightarrow V_{k}\right)=0\right\} \\
& =\left\{V \in \bmod J_{\sigma} \mid \operatorname{Hom}\left(V, s_{k}\right)=0\right\} \\
& =:\left(\bmod J_{\sigma}\right)^{k},
\end{aligned}
$$

$\bmod J_{\sigma} \cap \Phi_{k}^{-1}\left(\bmod J_{\sigma^{\prime}}\right)[1]=\left\{V \in \bmod J_{\sigma} \mid V_{i}=0(i \neq k)\right\}$

$$
=: \mathcal{S}_{k} \text {. }
$$


In other words, $\left(\left(\bmod J_{\sigma}\right)^{k}, \mathcal{S}_{k}\right)$ is a torsion pair of $\bmod J_{\sigma}$, and $\Phi_{k}^{-1}\left(\bmod J_{\sigma^{\prime}}\right)$ is obtained from $\bmod J_{\sigma}$ by tilting with respect to this torsion pair (see [Nagao 2013, §3.1]). Then we have

$$
\begin{aligned}
\bmod J_{\sigma^{\prime}} \cap \Phi_{k}\left(\bmod J_{\sigma}\right) & =\left\{V \in \bmod J_{\sigma^{\prime}} \mid \operatorname{Hom}\left(s_{k}^{\prime}, V\right)=0\right\} \\
& =:\left(\bmod J_{\sigma^{\prime}}\right)_{k} .
\end{aligned}
$$

In summary, we have the following:

Proposition 2.3. The equivalence $\Phi_{k}$ induces an equivalence of $\left(\bmod J_{\sigma}\right)^{k}$ and $\left(\bmod J_{\sigma^{\prime}}\right)_{k}$.

Nagao [2012, proof of Proposition 3.1] gave the isomorphism in Theorem 2.2(1) explicitly. For $V \in \bmod J_{\sigma} \cap \Phi_{k}^{-1}\left(\bmod J_{\sigma^{\prime}}\right)$, the map

$$
\left(H_{\bmod J_{\sigma^{\prime}}}^{0}\left(\Phi_{k}(V)\right)\right)_{k-1} \rightarrow\left(H_{\bmod J_{\sigma^{\prime}}}^{0}\left(\Phi_{k}(V)\right)\right)_{k}
$$

is induced by the morphism

$$
R_{k-1} \oplus R_{k-1, k+1}: V_{k-1} \rightarrow V_{k-1} \oplus V_{k+1},
$$

where

and

$$
R_{k-1}:= \begin{cases}r_{k-1} & k-1 \in \widehat{I}_{r}, \\ h_{k-\frac{3}{2}}^{+} \circ h_{k-\frac{3}{2}}^{-} & k-1 \notin \widehat{I}_{r},\end{cases}
$$

$$
R_{k-1, k+1}:=h_{k+\frac{1}{2}}^{-} \circ h_{k+\frac{1}{2}}^{+} \circ h_{k-\frac{1}{2}}^{+} .
$$

2D. Cut and mutation. Let $(Q, W)$ be a quiver with potential. To each subset $C \subset Q_{1}$, we associate a grading $g_{C}$ on $Q$ by

$$
g_{C}(a)= \begin{cases}1 & a \in C \\ 0 & a \in C .\end{cases}
$$

A subset $C \subset Q_{1}$ is called a cut if $W$ is homogeneous of degree 1 with respect to $g_{C}$. Denote by $Q_{C}$ the subquiver of $Q$ with vertex set $Q_{0}$ and arrow set $Q_{1} \backslash C$. We define the truncated Jacobian algebra by

$$
J(Q, W)_{C}:=J(Q, W) /\langle C\rangle .
$$

Let $k$ be a vertex of $Q_{\sigma}$ without loops and $C$ be a cut of $\left(Q_{\sigma}, w_{\sigma}\right)$ such that $g_{C}\left(h_{k+\frac{1}{2}}^{+}\right)=1^{4}$. We define a cut $C^{\prime}$ of $\left(Q_{\sigma^{\prime}}, w_{\sigma^{\prime}}\right)$ by the following conditions:

\footnotetext{
${ }^{4}$ We can construct a cut of $\left(Q_{\sigma}, w_{\sigma}\right)$ as follows: First, by coupling $h_{i}^{+}$and $h_{i}^{-}$for each $i$, we group the arrows in $Q_{\sigma}$ into $N+\left|\widehat{I}_{r}\right|$ groups. Note that $N+\left|\widehat{I}_{r}\right|$ is even. These groups have the natural cyclic order and we label each of them as odd or even. Choose (any) one arrow from each odd (or even) labeled group; then we get a cut.
} 
- $g_{C^{\prime}}\left(h_{k-\frac{1}{2}}^{+}\right)=1$.

- $g_{C^{\prime}}\left(h_{i}^{ \pm}\right)=g_{C}\left(h_{i}^{ \pm}\right)$if $i \neq k-\frac{1}{2}, k+\frac{1}{2}$.

Proposition 2.4 [Nagao 2011c, Proposition 4.12]. The equivalence $\Phi_{k}$ induces an equivalence of $\left(\bmod J_{\sigma, C}\right)_{k}$ and $\left(\bmod J_{\sigma^{\prime}, C^{\prime}}\right)^{k}$.

Proof. It is enough to show that if $h_{k+\frac{1}{2}}^{+}$vanishes on $V$, then $h_{k-\frac{1}{2}}^{+}$vanished on $\Phi_{k}(V)$.

Since $g_{C}\left(h_{k-\frac{1}{2}}^{ \pm}\right)=0$, we have

- $g_{C}\left(r_{k-1}\right)=1$ if $k-1 \in \widehat{I}_{r}$, and

- $g_{C}\left(h_{k-\frac{3}{2}}^{+}\right)=1$ or $g_{C}\left(h_{k-\frac{3}{2}}^{-}\right)=1$ if $k-1 \notin \widehat{I}_{r}$,

and so $R_{k-1}$ vanishes. Since $g_{C}\left(h_{k+\frac{1}{2}}^{+}\right)=1$, we see that $R_{k-1, k+1}$ vanishes.

\section{Motivic Donaldson-Thomas invariants}

3A. Motives. We are working in a version of the ring of motivic weights: let $\mathcal{M}_{\mathbb{C}}$ denote the $K$-group of the category of effective Chow motives over $\mathbb{C}$, extended by $\mathbb{L}^{-\frac{1}{2}}$, where $\mathbb{L}$ is the Lefschetz motive. It has a natural structure of a $\lambda$-ring [Getzler 1996; Heinloth 2007], with $\sigma$-operations defined by $\sigma_{n}([X])=\left[X^{n} / S_{n}\right]$ and $\sigma_{n}\left(\mathbb{L}^{\frac{1}{2}}\right)=\mathbb{L}^{\frac{n}{2}}$. We put

$$
\tilde{\mathcal{M}}_{\mathbb{C}}=\mathcal{M}_{\mathbb{C}} \llbracket \mathbb{L}^{-1} \rrbracket,
$$

which is also a $\lambda$-ring. Note that in this latter ring the elements $\left(1-\mathbb{L}^{n}\right)$, and therefore the motives of general linear groups, are invertible. The rings $\mathcal{M}_{\mathbb{C}} \subset \tilde{\mathcal{M}}_{\mathbb{C}}$ sit in larger rings $\mathcal{M}_{\mathbb{C}}^{\hat{\mu}} \subset \tilde{\mathcal{M}}_{\mathbb{C}}^{\hat{\mu}}$ of equivariant motives, where $\hat{\mu}$ is the group of all roots of unity [Looijenga 2002].

Let $f: X \rightarrow \mathbb{C}$ be a regular function on a smooth variety $X$. Using arc spaces, Denef and Loeser [2001; Looijenga 2002] defined the motivic nearby cycle $\left[\psi_{f}\right] \in$ $\mathcal{M}_{\mathbb{C}}^{\hat{\mu}}$ and the motivic vanishing cycle

$$
\left[\varphi_{f}\right]:=\left[\psi_{f}\right]-\left[f^{-1}(0)\right] \in \mathcal{M}_{\mathbb{C}}^{\hat{\mu}}
$$

of $f$. Note that if $f=0$, then $\left[\varphi_{0}\right]=-[X]$.

Theorem 3.1 [Behrend et al. 2013, Proposition 1.11]. Let $f: X \rightarrow \mathbb{C}$ be a regular function on a smooth variety $X$. Assume that $X$ admits a $\mathbb{C}^{*}$-action such that $f$ is $\mathbb{C}^{*}$-equivariant, i.e., $f(t x)=\operatorname{tf}(x)$ for $t \in \mathbb{C}^{*}, x \in X$, and such that there exist limits $\lim _{t \rightarrow 0}$ t $x$ for all $x \in X$. Then

$$
\left[\varphi_{f}\right]=\left[f^{-1}(1)\right]-\left[f^{-1}(0)\right] \in \mathcal{M}_{\mathbb{C}} \subset \mathcal{M}_{\mathbb{C}}^{\hat{\mu}} .
$$


Following [Behrend et al. 2013], we define the virtual motive of $\operatorname{crit}(f)$ to be

$$
[\operatorname{crit}(f)]_{\mathrm{vir}}:=-\left(-\mathbb{L}^{\frac{1}{2}}\right)^{-\operatorname{dim} X}\left[\varphi_{f}\right] \in \mathcal{M}_{\mathbb{C}}^{\hat{\mu}} .
$$

For a smooth variety $X$, we put

$$
[X]_{\mathrm{vir}}:=\left[\operatorname{crit}\left(0_{X}\right)\right]_{\mathrm{vir}}=\left(-\mathbb{1}^{\frac{1}{2}}\right)^{-\operatorname{dim} X} \cdot[X] .
$$

3B. Quivers and moduli spaces. Let $Q$ be a quiver, with vertex set $Q_{0}$ and edge set $Q_{1}$. For an arrow $a \in Q_{1}$, we denote by $s(a) \in Q_{0}$ and $t(a) \in Q_{0}$ the vertices at which $a$ starts and ends, respectively. We define the Euler-Ringel form $\chi$ on $\mathbb{Z}^{Q_{0}}$ by the rule

$$
\chi(\alpha, \beta)=\sum_{i \in Q_{0}} \alpha_{i} \beta_{i}-\sum_{a \in Q_{1}} \alpha_{s(a)} \beta_{t(a)}, \quad \alpha, \beta \in \mathbb{Z}^{Q_{0}} .
$$

Given a $Q$-representation $M$, we define its dimension vector $\underline{\operatorname{dim}} M \in \mathbb{N} Q_{0}$ by $\underline{\operatorname{dim}} M=\left(\operatorname{dim} M_{i}\right)_{i \in Q_{0}}$. Let $\alpha \in \mathbb{N}^{Q_{0}}$ be a dimension vector and let $V_{i}=\mathbb{C}^{\alpha_{i}}$ for $i \in Q_{0}$. We define

$$
R(Q, \alpha):=\bigoplus_{a \in Q_{1}} \operatorname{Hom}\left(V_{s(a)}, V_{t(a)}\right)
$$

and

$$
G_{\alpha}:=\prod_{i \in Q_{0}} \mathrm{GL}\left(V_{i}\right)
$$

Note that $G_{\alpha}$ naturally acts on $R(Q, \alpha)$, and the quotient stack

$$
\mathfrak{M}(Q, \alpha):=\left[R(Q, \alpha) / G_{\alpha}\right]
$$

gives the moduli stack of representations of $Q$ with dimension vector $\alpha$.

Let $W$ be a potential on $Q$, i.e., a finite linear combination of cyclic paths in $Q$. Denote by $J=J_{Q, W}$ the Jacobian algebra, i.e., the quotient of the path algebra $\mathbb{C} Q$ by the two-sided ideal generated by formal partial derivatives of the potential $W$. Let

$$
f_{\alpha}: R(Q, \alpha) \rightarrow \mathbb{C}
$$

be the $G_{\alpha}$-invariant function defined by taking the trace of the map associated to the potential $W$. As it is now well-known [Segal 2008, Proposition 3.8], a point in the critical locus $\operatorname{crit}\left(f_{\alpha}\right)$ corresponds to a $J$-module. The quotient stack

$$
\mathfrak{M}(J, \alpha):=\left[\operatorname{crit}\left(f_{\alpha}\right) / G_{\alpha}\right]
$$

gives the moduli stack of $J$-modules with dimension vector $\alpha$. 
Definition 3.2. A central charge is a group homomorphism $Z: \mathbb{Z}^{Q_{0}} \rightarrow \mathbb{C}$ such that

$$
Z(\alpha) \in \mathbb{H}_{+}=\left\{r e^{i \pi \varphi} \mid r>0,0<\varphi \leq 1\right\}
$$

for any $\alpha \in \mathbb{N} Q_{0} \backslash\{0\}$. Given $\alpha \in \mathbb{N} Q_{0} \backslash\{0\}$, the number $\varphi(\alpha)=\varphi \in(0,1]$ such that $Z(\alpha)=r e^{i \pi \varphi}$, for some $r>0$, is called the phase of $\alpha$.

Definition 3.3. For any nonzero $Q$-representation or $J$-module $V$, we define $\varphi(V)=\varphi(\operatorname{dim} V)$. A $Q$-representation (resp. $J$-module) $V$ is said to be $Z$-stable if for any proper nonzero $Q$-subrepresentation (resp. $J$-submodule) $U \subset V$ we have

$$
\varphi(U)<\varphi(V),
$$

and $Z$-semistable if for all such proper subrepresentations (resp. submodules) we have the weaker condition

$$
\varphi(U) \leq \varphi(V) .
$$

Definition 3.4. Given $\zeta \in \mathbb{R}^{Q_{0}}$, define the central charge $Z: \mathbb{Z}^{Q_{0}} \rightarrow \mathbb{C}$ by the rule

$$
Z(\alpha)=-\zeta \cdot \alpha+i|\alpha|,
$$

where $|\alpha|=\sum_{i \in Q_{0}} \alpha_{i}$. We call a $Q$-representation or $J$-module $\zeta$-(semi)stable if it is $Z$-(semi)stable.

Remark 3.5. Let the central charge $Z$ be as in Definition 3.4. Define the slope function $\mu: \mathbb{N} Q_{0} \backslash\{0\} \rightarrow \mathbb{R}$ by $\mu(\alpha)=\zeta \cdot \alpha /|\alpha|$. If $l \subset \mathbb{N}=\mathbb{M}+\cup\{0\}$ is a ray such that $Z(\alpha) \in l$, then $l=\mathbb{R}_{\geq 0}(-\mu(\alpha), 1)$. This implies that $\varphi(\alpha)<\varphi(\beta)$ if and only if $\mu(\alpha)<\mu(\beta)$.

We say that $\zeta \in \mathbb{R}^{Q_{0}}$ is $\alpha$-generic if for any $0<\beta<\alpha$ we have $\varphi(\beta) \neq \varphi(\alpha)$. This condition implies that any $\zeta$-semistable $Q$-representation or $J$-module is automatically $\zeta$-stable.

Let $R_{\zeta}(Q, \alpha)$ denote the open subset of $R(Q, \alpha)$ consisting of $\zeta$-semistable representations. Let $f_{\zeta, \alpha}$ denote the restriction of $f_{\alpha}$ to $R_{\zeta}(Q, \alpha)$. The quotient stacks

$$
\mathfrak{M}_{\zeta}(Q, \alpha):=\left[R_{\zeta}(Q, \alpha) / G_{\alpha}\right] \quad \text { and } \mathfrak{M}_{\zeta}(J, \alpha):=\left[\operatorname{crit}\left(f_{\zeta, \alpha}\right) / G_{\alpha}\right]
$$

give the moduli stacks of $\zeta$-semistable $Q$-representations and $J$-modules with dimension vector $\alpha$.

3C. Motivic DT invariants. Let $(Q, W)$ be a quiver with a potential and let $J=$ $J_{Q, W}$ be its Jacobian algebra. Recall that the degeneracy locus of the function $f_{\alpha}: R(Q, \alpha) \rightarrow \mathbb{C}$ defines the locus of $J$-modules, so that the quotient stack

$$
\mathfrak{M}(J, \alpha):=\left[\operatorname{crit}\left(f_{\alpha}\right) / G_{\alpha}\right]
$$


is the stack of $J$-modules with dimension vector $\alpha$. We define motivic DonaldsonThomas invariants by

$$
[\mathfrak{M}(J, \alpha)]_{\mathrm{vir}}:=\frac{\left[\operatorname{crit}\left(f_{\alpha}\right)\right]_{\mathrm{vir}}}{\left[G_{\alpha}\right]_{\mathrm{vir}}} .
$$

For a stability parameter $\zeta$, we define

$$
\left[\mathfrak{M}_{\zeta}(J, \alpha)\right]_{\mathrm{vir}}=\frac{\left[\operatorname{crit}\left(f_{\zeta, \alpha}\right)\right]_{\mathrm{vir}}}{\left[G_{\alpha}\right]_{\mathrm{vir}}},
$$

where, as before, $f_{\zeta, \alpha}$ denotes the restriction of $f_{\alpha}: R(Q, \alpha) \rightarrow \mathbb{C}$ to $R_{\zeta}(Q, \alpha)$.

3D. Generating series of motivic DT invariants. Let $(Q, W)$ be a quiver with a potential admitting a cut, and let $J=J_{Q, W}$ be its Jacobian algebra.

Definition 3.6. We define the generating series of the motivic Donaldson-Thomas invariants of $(Q, W)$ by

$$
A_{U}(y)=\sum_{\alpha \in \mathbb{N} Q_{0}}[\mathfrak{M}(J, \alpha)]_{\text {vir }} \cdot y^{\alpha}=\sum_{\alpha \in \mathbb{N} Q_{0}} \frac{\left[\operatorname{crit}\left(f_{\alpha}\right)\right]_{\text {vir }}}{\left[G_{\alpha}\right]_{\text {vir }}} \cdot y^{\alpha} \in \mathcal{T}_{Q},
$$

where the subscript refers to the fact that we think of this series as the universal series.

Given a cut $C$ of $(Q, W)$, we define a new quiver $Q_{C}=\left(Q_{0}, Q_{1} \backslash C\right)$. Let $J_{C}$ be the quotient of $\mathbb{C} Q_{C}$ by the ideal

$$
\left(\partial_{C} W\right)=(\partial W / \partial a, a \in C) .
$$

Proposition 3.7 [Morrison et al. 2012, Proposition 1.14]. If $(Q, W)$ admits a cut $C$, then

$$
A_{U}(y)=\sum_{\alpha \in \mathbb{N} Q_{0}}\left(-\mathbb{L}^{\frac{1}{2}}\right)^{\chi(\alpha, \alpha)+2 d_{I}(\alpha)} \frac{\left[R\left(J_{C}, \alpha\right)\right]}{\left[G_{\alpha}\right]} y^{\alpha},
$$

where $d_{C}(\alpha)=\sum_{(a: i \rightarrow j) \in C} \alpha_{i} \alpha_{j}$ for any $\alpha \in \mathbb{Z}^{Q_{0}}$.

The quiver with potential $\left(Q_{\sigma}, w_{\sigma}\right)$ introduced in Section 2 admits a cut (see Section 2D), and Proposition 3.7 can be applied. In the next section we use this to compute the universal series in a specific case.

\section{The universal DT series: special case}

Throughout this section we fix $\sigma$ to be the unique partition defined such that

$$
\widehat{I}_{r}=\left\{0,1,2,3, \ldots, N^{\prime}-1\right\},
$$

in other words, the partition such that the quiver with potential $\left(Q_{\sigma}, w_{\sigma}\right)$ has loops at the first $N^{\prime}$ vertices only.

The aim of this section is to prove Theorem 0.1 for this quiver with potential. 


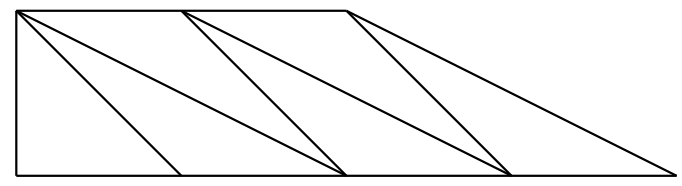

Figure 6. The case $N=6$ and $N^{\prime}=2$.

We define three fixed subsets of the vertices:

$$
\begin{aligned}
& I_{1}:=\left\{0,1, \ldots, N^{\prime}-1\right\} \subset \mathbb{Z} / N, \\
& I_{2}:=\left\{N^{\prime}, N^{\prime}+2, N^{\prime}+4, \ldots, N-2\right\} \subset \mathbb{Z} / N, \\
& I_{3}:=\left\{N^{\prime}+1, N^{\prime}+3, N^{\prime}+5, \ldots, N-1\right\} \subset \mathbb{Z} / N .
\end{aligned}
$$

Then there exists a cut $C$ given by the collection of arrows

$$
C=\left\{h_{i}^{-} \mid i-\frac{1}{2} \notin I_{2}\right\} .
$$

By Proposition 3.7, the universal DT series $A_{U}^{\sigma}(y)=\sum_{\alpha \in \mathbb{N} Q} A_{\alpha} y^{\alpha}$ has coefficients given by

$$
A_{\alpha}=\left(-\mathbb{L}^{\frac{1}{2}}\right)^{\chi(\alpha, \alpha)+2 d_{C}(\alpha)} \frac{\left[R\left(J_{\sigma, C}, \alpha\right)\right]}{\left[G_{\alpha}\right]} y^{\alpha},
$$

where $d_{C}(\alpha)=\sum_{(a: i \rightarrow j) \in C} \alpha_{i} \alpha_{j}$. To begin, we find a simple expression for the term $\chi(\alpha, \alpha)+2 d_{C}(\alpha)$ in the exponent. We know by definition that

$$
\begin{aligned}
\chi(\alpha, \alpha) & =\sum_{i \in I_{1} \cup I_{2} \cup I_{3}} \alpha_{i}^{2}-\sum_{i \in I_{1}} \alpha_{i}^{2}-\sum_{i \in I_{1} \cup I_{2} \cup I_{3}} \alpha_{i} \alpha_{i+1}-\sum_{i \in I_{1} \cup I_{2} \cup I_{3}} \alpha_{i+1} \alpha_{i}, \\
d_{I}(\alpha) & =\sum_{i \in I_{1}} \alpha_{i} \alpha_{i+1}+\sum_{i \in I_{3}} \alpha_{i+1} \alpha_{i}
\end{aligned}
$$

so it follows that

$$
\chi(\alpha, \alpha)+2 d_{C}(\alpha)=\sum_{i \in I_{2} \cup I_{3}} \alpha_{i}^{2}-2 \cdot \sum_{i \in I_{2}} \alpha_{i} \alpha_{i+1}=\sum_{i \in I_{2}}\left(\alpha_{i+1}-\alpha_{i}\right)^{2} .
$$

Our next goal is to factorize $A_{U}^{\sigma}(y)$ into two simpler series. We proceed by analyzing the motivic classes $\left[R\left(J_{\sigma, C}, \alpha\right)\right]$.

Given a dimension vector $\alpha \in \mathbb{N} Q_{0}$ and a representation of a $J_{\sigma, C}$-module

$$
V=\bigoplus_{i \in I_{1} \cup I_{2} \cup I_{3}} V_{i}
$$

we focus on the specific element

$$
H:=h_{\frac{1}{2}}^{+}+h_{\frac{3}{2}}^{+}+\cdots+h_{N-\frac{1}{2}}^{+} \in \bigoplus_{i \in I_{1} \cup I_{2} \cup I_{3}} \operatorname{Hom}\left(V_{i}, V_{i+1}\right) .
$$


This map $H$ acts as an endomorphism of the vector space $V$. Given any such linear map

$$
H: V \rightarrow V,
$$

there exists a unique splitting $V=V^{I} \oplus V^{N}$ with maps

$$
\begin{gathered}
H^{I}: V^{I} \rightarrow V^{I} \text { invertible, } \\
H^{N}: V^{N} \rightarrow V^{N} \text { nilpotent, }
\end{gathered}
$$

so that

$$
H=H^{I} \oplus H^{N} .
$$

Moreover, in our case the above splitting respects the grading by $i \in I_{1} \cup I_{2} \cup I_{3}$. To be explicit, we have that

$$
V^{I}=\bigoplus_{i \in I_{1} \cup I_{2} \cup I_{3}} V_{i}^{I}
$$

where $V_{i}^{I}:=V_{i} \cap V^{I}$ (similarly $V^{N}=\bigoplus_{i \in I_{1} \cup I_{2} \cup I_{3}} V_{i}^{N}$ with $V_{i}^{N}:=V_{i} \cap V^{N}$ ). One immediate consequence of this is that

$$
\operatorname{dim}\left(V_{i}^{I}\right)=\operatorname{dim}\left(V_{i+1}^{I}\right) \text { for all } i \in I_{1} \cup I_{2} \cup I_{3} ;
$$

indeed, this is clear since the block form of $H^{I}$ demands that it map $V_{i}^{I}$ to $V_{i+1}^{I}$ via an isomorphism. We are now ready to decompose the computation of $A_{U}^{\sigma}(y)$ into two simpler subproblems.

Definition 4.1 (invertible series). We define

$$
R^{I}(a):=\left\{r \in R\left(J_{\sigma, C}, \alpha\right) \mid H \text { is invertible, } \alpha_{i}=a \text { for all } i\right\}
$$

and the series

$$
I^{\sigma}(x):=\sum_{a \geq 0} \frac{\left[R^{I}(a)\right]}{[\operatorname{GL}(a)]^{N}} x^{a} .
$$

Definition 4.2 (nilpotent series). We define

$$
R^{N}(\alpha):=\left\{r \in R\left(J_{\sigma, C}, \alpha\right) \mid H \text { is nilpotent }\right\}
$$

and the series

$$
N^{\sigma}(y):=\sum_{\alpha \in \mathbb{N} Q_{0}}\left(-\mathbb{L}^{\frac{1}{2}}\right)^{\sum_{i \in I_{2}}\left(\alpha_{i+1}-\alpha_{i}\right)^{2}} \frac{\left[R^{N}(\alpha)\right]}{\left[G_{\alpha}\right]} y^{\alpha} .
$$

The following lemma shows that the series $A_{U}^{\sigma}(y)$ factorizes into the product of the two series just defined: 
Lemma 4.3. Let $x=y_{0} \cdots y_{N-1}$; then, in the notation above, we have

$$
A_{U}^{\sigma}(y)=I^{\sigma}(x) \cdot N^{\sigma}(y) .
$$

Proof. This formula follows directly from a stratification of the variety $R\left(J_{\sigma, C}, \alpha\right)$ by the dimension of $V_{i}^{I}$.

Fix $\alpha \in \mathbb{N}^{Q_{0}}$; we stratify $R\left(J_{\sigma, C}, \alpha\right)$ by $\operatorname{dim}\left(V_{i}^{I}\right)=a$. Let

$$
\underline{a}:=(a, a, \ldots, a) \in \mathbb{N}^{Q_{0}},
$$

and let

$$
\alpha^{\prime} \text { be such that } \alpha=\underline{a}+\alpha^{\prime} \in \mathbb{N}^{Q_{0}} .
$$

There is a Zariski locally trivial fibration

$$
R^{I}(a) \times R^{N}\left(\alpha^{\prime}\right) \longrightarrow\left\{r \in R\left(J_{\sigma, C}, \alpha\right) \mid \operatorname{dim}\left(V_{i}^{I}\right)=a \text { for } H \in r\right\}
$$

Here $\mathcal{M}(a, \alpha)$ is the space parametrizing splittings $V_{i}=V_{i}^{I} \oplus V_{i}^{N}$. To see this, one checks that the arrows $r_{i}, h_{i+\frac{1}{2}}^{-}$in the representation also preserve the splitting, so the entire representation splits into $V^{I} \oplus V^{N}$. This follows easily from the relations and some basic linear algebra.

Splittings of the vector space $V_{i}=V_{i}^{I} \oplus V_{i}^{N}$ are parametrized by

$$
\operatorname{GL}\left(\alpha_{i}\right) /\left(\operatorname{GL}(a) \times \mathrm{GL}\left(\alpha_{i}^{\prime}\right)\right),
$$

and hence the motivic class of the base is

$$
[\mathcal{M}(a, \alpha)]=\frac{\left[G_{\alpha}\right]}{[\mathrm{GL}(a)]^{N} \cdot\left[G_{\alpha^{\prime}}\right]} .
$$

Summing over each stratum with $\operatorname{dim}\left(V_{i}^{I}\right)=a$, we get

$$
\left[R\left(J_{\sigma, C}, \alpha\right)\right]=\left[G_{\alpha}\right] \cdot \sum_{a=0}^{\min _{i}\left\{\alpha_{i}\right\}} \frac{\left[R^{I}(a)\right]}{[\mathrm{GL}(a)]^{N}} \cdot \frac{\left[R^{N}\left(\alpha^{\prime}\right)\right]}{\left[G_{\alpha^{\prime}}\right]} .
$$

Multiplying both sides of this expression by $\left(-\llbracket^{\frac{1}{2}}\right)^{\sum_{i \in I_{2}}\left(\alpha_{i+1}-\alpha_{i}\right)^{2}} y^{\alpha}$ and summing gives

$$
\begin{aligned}
& A_{U}^{\sigma}(y) \\
& \quad=\left(\sum_{a \geq 0} \frac{\left[R^{I}(a)\right]}{[\mathrm{GL}(a)]^{N}} \prod_{i=0}^{N-1} y_{i}^{a}\right) \cdot\left(\sum_{\alpha^{\prime} \in \mathbb{N} Q_{0}}\left(-\mathbb{L}^{\frac{1}{2}}\right)^{\sum_{i \in I_{2}}\left(\alpha_{i+1}^{\prime}-\alpha_{i}^{\prime}\right)^{2}} \frac{\left[R^{N}\left(\alpha^{\prime}\right)\right]}{\left[G_{\alpha^{\prime}}\right]} y^{\alpha^{\prime}}\right),
\end{aligned}
$$

proving the result. 
In the next two sections we compute formulae for $I^{\sigma}(x)$ and $N^{\sigma}(y)$.

4A. Step I: the invertible case $I^{\sigma}(x)$.

Proposition 4.4. We have

$$
I^{\sigma}(x)=\operatorname{Exp}\left(\mathbb{R} \frac{x}{1-x}\right) .
$$

Proof. A $J_{\sigma, C}$-module $r \in R\left(J_{\sigma, C}, \alpha\right)$ is given by a vector space

$$
V=\bigoplus_{i \in I_{1} \cup I_{2} \cup I_{3}} V_{i}
$$

of dimension $\alpha \in \mathbb{N} Q_{0}$ and a collection of linear maps

$$
\begin{array}{cl}
r_{i}: V_{i} \rightarrow V_{i} & \text { for } i \in I_{1}, \\
h_{i+\frac{1}{2}}^{-}: V_{i+1} \rightarrow V_{i} & \text { for } i \in I_{2}, \\
h_{i+\frac{1}{2}}^{+}: V_{i} \rightarrow V_{i+1} & \text { for } i \in I_{1} \cup I_{2} \cup I_{3},
\end{array}
$$

satisfying the relations coming from cyclic differentiation of the potential

$$
\begin{aligned}
r_{i} h_{i-\frac{1}{2}}^{+} & =h_{i-\frac{1}{2}}^{+} r_{i-1} & & \text { for } i \in\left[1, N^{\prime}-1\right] \cap I_{1}, \\
r_{0} h_{N-\frac{1}{2}}^{+} & =h_{N-\frac{1}{2}}^{+} h_{N-\frac{3}{2}}^{+} h_{N-\frac{3}{2}}^{-}, & & \\
h_{N^{\prime}+\frac{1}{2}}^{-} h_{N^{\prime}+\frac{1}{2}}^{+} h_{N^{\prime}-\frac{1}{2}}^{+} & =h_{N^{\prime}-\frac{1}{2}}^{+} r_{N^{\prime}-1}, & & \\
h_{i+\frac{3}{2}}^{-} h_{i+\frac{3}{2}}^{+} h_{i+\frac{1}{2}}^{+} & =h_{i+\frac{1}{2}}^{+} h_{i-\frac{1}{2}}^{+} h_{i-\frac{1}{2}}^{-} & & \text {for } i=\left[N^{\prime}+1, N-3\right] \cap I_{3} .
\end{aligned}
$$

Assuming moreover that $r \in R^{I}(a)$, we have

$$
h_{i+\frac{1}{2}}^{+}: V_{i} \rightarrow V_{i+1} \text { is invertible for all } i \in I_{1} \cup I_{2} \cup I_{3} \text {. }
$$

This allows us to express $R^{I}(a)$ as a $\prod_{i=1}^{N-1} \operatorname{GL}\left(V_{i}\right)$-torsor over a commuting variety

$$
\begin{aligned}
\pi: R^{I}(a) & \rightarrow C(a), \\
\left(r_{i}, h_{i+\frac{1}{2}}^{+}, h_{i+\frac{1}{2}}^{-}\right) & \mapsto\left(r_{0}, h_{N-\frac{1}{2}}^{+} h_{N-\frac{3}{2}}^{+} \cdots h_{\frac{3}{2}}^{+} h_{\frac{1}{2}}^{+}\right),
\end{aligned}
$$

where

$$
C(a)=\left\{(A, B) \in \operatorname{End}\left(V_{0}\right) \times \mathrm{GL}\left(V_{0}\right) \mid A B=B A\right\} .
$$


The free action of $\prod_{i=1}^{N-1} \mathrm{GL}\left(V_{i}\right)$ on $R^{I}(a)$ is given by

$$
\begin{array}{rlrl}
\left(g_{1}, \ldots, g_{N-1}\right): r_{i} & \mapsto g_{i} r_{i} g_{i}^{-1} & \\
h_{\frac{1}{2}}^{+} \mapsto g_{1} h_{\frac{1}{2}}^{+}, & \\
h_{N-\frac{1}{2}}^{+} & \mapsto h_{N-\frac{1}{2}}^{+} g_{N-1}^{-1}, & \\
h_{i+\frac{1}{2}}^{+} & \mapsto g_{i+1} h_{i+\frac{1}{2}}^{+} g_{i}^{-1} & \text { for } i \in\left[1, N^{\prime}-1\right], \\
h_{i+\frac{1}{2}}^{-} & \mapsto g_{i} h_{i+\frac{1}{2}}^{-} g_{i+1}^{-1} & \text { for } i \in[1, N-2],
\end{array}
$$

As GL(a) is a special group [Chevalley et al. 1958], the torsor splits in the Zariski topology, so motivically we have

$$
\left[R^{I}(a)\right]=[\mathrm{GL}(a)]^{N-1} \cdot[C(a)]
$$

Thus

$$
I^{\sigma}(x)=\sum_{a \geq 0} \frac{[C(a)]}{[\mathrm{GL}(a)]} x^{a}
$$

The generating series for the commuting variety is obtained in [Bryan and Morrison 2015], giving the result.

4B. Step II: the nilpotent case $N^{\sigma}(y)$. This section is the final step in the calculation. Here we compute $N^{\sigma}(y)$ and obtain the formula of $A_{U}^{\sigma}(y)$.

We fix a dimension vector $\alpha \in \mathbb{N} Q_{0}$. As before, a $J_{\sigma, C}$-module is given by a vector space

$$
V=\bigoplus_{i \in I_{1} \cup I_{2} \cup I_{3}} V_{i}
$$

of dimension $\alpha$ and a collection of linear maps

$$
\begin{array}{cl}
r_{i}: V_{i} \rightarrow V_{i} & \text { for } i \in I_{1}, \\
h_{i+\frac{1}{2}}^{-}: V_{i+1} \rightarrow V_{i} & \text { for } i \in I_{2}, \\
h_{i+\frac{1}{2}}^{+}: V_{i} \rightarrow V_{i+1} & \text { for } i \in I_{1} \cup I_{2} \cup I_{3},
\end{array}
$$

satisfying the relations of the potential (see Proposition 4.4). Throughout this section we insist that the map

$$
H=h_{\frac{1}{2}}^{+}+h_{\frac{3}{2}}^{+}+\cdots+h_{N-\frac{1}{2}}^{+} \in \bigoplus_{i \in I_{1} \cup I_{2} \cup I_{3}} \operatorname{Hom}\left(V_{i}, V_{i+1}\right)
$$


is nilpotent. In fact, $R^{N}(\alpha)$ is exactly the collection of all such representations (see Definition 4.2). In particular, if we let $|\alpha|:=\operatorname{dim}(V)$ then we know that $H^{|\alpha|}=0$. This gives a filtration of the vector space

$$
V=V^{|\alpha|} \supset V^{|\alpha|-1} \supset \cdots \supset V^{1} \supset V^{0}=\{0\},
$$

where

$$
V^{j}=\left\{v \in V \mid H^{j}(v)=0\right\} .
$$

Moreover, the filtration respects the grading by $i \in I_{1} \cup I_{2} \cup I_{3}$, by which we mean that

$$
V^{j}=\bigoplus_{i \in I_{1} \cup I_{2} \cup I_{3}}\left(V^{j} \cap V_{i}\right)
$$

where $V_{i}$ is the summand at the $i$-th vertex of the quiver. By considering the vector space $V$ as a representation of the nilpotent matrix $H$, we can identify $V$ with a $\mathbb{C}[x]$-module supported at the origin. Modules for a principal ideal domain have a simple structure. In particular, we have

$$
V \cong \bigoplus_{j=1}^{d}\left(\mathbb{C}[x] /\left(x^{j}\right)\right)^{\oplus b_{j}}
$$

as a $\mathbb{C}[x]$-module. The next proposition provides a more refined version of this statement, where each factor in this decomposition is generated by a vector from a vector space $V_{i}$ :

Proposition 4.5. For each $i \in I_{1} \cup I_{2} \cup I_{3}$, there exists a collection of integers $b_{j}^{i}$ such that

$$
V \cong \bigoplus_{i \in I_{1} \cup I_{2} \cup I_{3}} \bigoplus_{i=1}^{d}\left(\mathbb{C}[x] /\left(x^{j}\right)\right)^{\oplus b_{j}^{i}}
$$

where the factor $\left(\mathbb{C}[x] /\left(x^{j}\right)\right)^{\oplus b_{j}^{i}}$ is generated as a $\mathbb{C}[x]$-module by vectors in $V_{i}$. Moreover, the numbers $b_{j}^{i}$ are uniquely determined by the above conditions.

Proof. We will argue by induction on $d$, the largest integer such that $b_{d} \neq 0$. As such, we can assume that for each $j \leq d-1$ the factor $\mathbb{C}[x] /\left(x^{j}\right)$ is generated by a vector in some $V_{i}$. Now let $e_{1}, \ldots, e_{b_{d}}$ be a generating set for the factor $\left(\mathbb{C}[x] /\left(x^{d}\right)\right)^{\oplus b_{d}}$, and define $W:=\operatorname{span}\left\{e_{1}, \ldots, e_{b_{d}}\right\}$. We consider the projection operators

$$
p_{i}: V \rightarrow V_{i} / V_{i} \cap V^{d-1},
$$

and set $W_{i}:=p_{i}(W)$ and $b_{d}^{i}=\operatorname{dim} W_{i}$. We claim that

$$
p_{0} \oplus \cdots \oplus p_{N-1}: W \rightarrow W_{0} \oplus \cdots \oplus W_{N-1}
$$


is an isomorphism. The map is clearly onto and an injection since any vector in the kernel must lie in $V^{d-1}$. Now, considering a lifting of the vector space $V_{i} \supset W_{i}^{\prime} \rightarrow W_{i} \subset V_{i} / V_{i} \cap V^{d-1}$, we have that

$$
W_{i}^{\prime} \oplus H W_{i}^{\prime} \oplus \cdots \oplus H^{d-1} W_{i}^{\prime} \subset V
$$

is a submodule of $V$ isomorphic to $\left(\mathbb{C}[x] /\left(x^{d}\right)\right)^{\oplus b_{d}^{i}}$. Summing over all $i$, we have that $\left(\mathbb{C}[x] /\left(x^{d}\right)\right)^{\sum_{i} b_{d}^{i}}$ is a submodule of $V$, and hence it follows that $\sum_{i} \operatorname{dim} W_{i}=$ $\sum_{i} b_{d}^{i} \leq b_{d}=\operatorname{dim} W$, and so for dimension reasons we get

$$
V \cong\left(\bigoplus_{i=0}^{N-1}\left(\mathbb{C}[x] /\left(x^{d}\right)\right)^{\oplus b_{d}^{i}}\right) \bigoplus\left(\bigoplus_{j=1}^{d-1}\left(\mathbb{C}[x] /\left(x^{j}\right)\right)^{\oplus b_{j}}\right) .
$$

Here each factor $\left(\mathbb{C}[x] /\left(x^{d}\right)\right)^{\oplus b_{d}^{i}}$ is generated by vectors in $V_{i}$, so by our inductive hypothesis the entire module is generated by vectors in $V_{i}$.

Finally we prove the uniqueness statement. Assume we have two distinct such decompositions

$$
V \cong \bigoplus_{i=0}^{N-1} \bigoplus_{j=1}^{d}\left(\mathbb{C}[x] /\left(x^{j}\right)\right)^{\oplus b_{j}^{i}} \cong \bigoplus_{i=0}^{N-1} \bigoplus_{j=1}^{d}\left(\mathbb{C}[x] /\left(x^{j}\right)\right)^{\oplus c_{j}^{i}}
$$

By restricting to subrepresentations if necessary, we can assume that $b_{d}^{i} \neq c_{d}^{i}$ for some $i$. However in this case

$$
b_{d}^{i}=\operatorname{dim}\left(\operatorname{ker}\left(H^{d}: V_{i} \rightarrow V_{i+d}\right) / V_{i} \cap V^{d-1}\right)=c_{d}^{i}
$$

is a contradiction. This proves the last part of the lemma.

Next we organize this data in the way most helpful to our cause:

Definition 4.6. Let $0 \leq a, b \leq N-1$. We define

$$
|b-a|=\min \{r \in\{0,1, \ldots, N-1\} \mid b=a+r \bmod N\} .
$$

Intuitively, this is the distance from $a$ to $b$ in the cyclic direction $i \rightarrow i+1$ corresponding to the map $H$.

Definition 4.7. Suppose we have a decomposition of $V$ as a $\mathbb{C}[x]$-module as in Proposition 4.5. Define $V^{a, b}$ to be the vector subspace corresponding to the summand

$$
\bigoplus_{l \geq 1}\left(\mathbb{C}[x] /\left(x^{N(l-1)+|b-a|+1}\right)\right)^{b_{N(l-1)+|b-a|+1}^{a},}
$$

and relabel the integers

$$
b_{l}^{a, b}:=b_{N(l-1)+|b-a|+1}^{a}
$$


to define partitions

$$
\pi^{[a, b]}:=\left(1^{b_{1}^{a, b}} 2^{b_{2}^{a, b}} 3^{b^{a, b}} \cdots\right) .
$$

Notice that the above definition depends on the choice of the decomposition in Proposition 4.5. However, all such vector spaces are isomorphic abstractly as $\mathbb{C}[x]$-modules. We can think of these vector spaces as being generated by the nilpotent vectors that start at the $a$-th vertex and are annihilated at the $(b+1)$-st vertex under the action of the map $H$.

The next lemma makes explicit how to recover the dimension vector of a representation from the datum of the $N^{2}$ partitions $\left\{\pi^{[a, b]} \mid 0 \leq a, b \leq N-1\right\}$.

Lemma 4.8. Given a representation $r \in R^{N}(\alpha)$ so that the endomorphism $H$ has type $\left\{\pi^{[a, b]}\right\}$, the dimension vector of the representation $r$ is given by

$$
\alpha_{i}=\sum_{a, b}\left|\pi^{[a, b]}\right|-\sum_{\substack{a, b \\ i \notin[a, b]}} l\left(\pi^{[a, b]}\right),
$$

where $\left|\pi^{[a, b]}\right|$ and $l\left(\pi^{[a, b]}\right)$ are the size and length of the partition $\pi^{[a, b]}$.

Proof. This is clear since

$$
V=\bigoplus_{a, b} V^{a, b}
$$

and

$$
\operatorname{dim}\left(V^{a, b} \cap V_{i}\right)= \begin{cases}\left|\pi^{[a, b]}\right| & \text { if } i \in[a, b], \\ \left|\pi^{[a, b]}\right|-l\left(\pi^{[a, b]}\right) & \text { if } i \notin[a, b] .\end{cases}
$$

We can use this to give a simple reformulation of the term $\chi(\alpha, \alpha)+2 d_{C}(\alpha)$ appearing in the series $N^{\sigma}$ :

Corollary 4.9. We have

$$
\chi(\alpha, \alpha)+2 d_{C}(\alpha)=\sum_{i \in I_{2}}\left(\sum_{b \neq i} l\left(\pi^{[i+1, b]}\right)-\sum_{c \neq i+1} l\left(\pi^{[c, i]}\right)\right)^{2} .
$$

Proof. In our initial analysis of these terms we saw that

$$
\chi(\alpha, \alpha)+2 d_{C}(\alpha)=\sum_{i \in I_{2}}\left(\alpha_{i+1}-\alpha_{i}\right)^{2},
$$

and now by Lemma 4.8 we have

$$
\alpha_{i+1}-\alpha_{i}=\sum_{b \neq i} l\left(\pi^{[i+1, b]}\right)-\sum_{c \neq i+1} l\left(\pi^{[c, i]}\right) .
$$

The above classification has been for the purpose of breaking the variety $R^{N}(\alpha)$ down into simpler pieces. 
Definition 4.10. Given $N^{2}$ partitions $\left\{\pi^{[a, b]} \mid 0 \leq a, b \leq N-1\right\}$ and a dimension vector $\alpha$ as in Lemma 4.8, we define

$$
R\left(\left\{\pi^{[a, b]}\right\}\right)=\left\{r \in R^{N}(\alpha) \mid H \text { has type }\left\{\pi^{[a, b]}\right\}\right\} .
$$

This provides a stratification of $R^{N}(\alpha)$ into strata where the normal form of $H$ has a fixed type. We will proceed to compute the motivic classes of each of these strata.

A representation in $R\left(\left\{\pi^{[a, b]}\right\}\right)$ is given explicitly by a vector space $V=$ $\bigoplus_{i \in I_{1} \cup I_{2} \cup I_{3}} V_{i}$ and a collection of linear maps corresponding to the arrows $r_{i}$ with $i \in I_{1}, h_{i+\frac{1}{2}}^{-}$with $i \in I_{2}$ and $h_{i+\frac{1}{2}}^{+}$with $i \in I_{1} \cup I_{2} \cup I_{3}$. In addition, the linear maps satisfy relations

$$
\begin{aligned}
r_{i} h_{i-\frac{1}{2}}^{+} & =h_{i-\frac{1}{2}}^{+} r_{i-1} & & \text { for } i \in\left[1, N^{\prime}-1\right] \cap I_{1}, \\
r_{0} h_{N-\frac{1}{2}}^{+} & =h_{N-\frac{1}{2}}^{+} h_{N-\frac{3}{2}}^{+} h_{N-\frac{3}{2}}^{-}, & & \\
h_{N^{\prime}+\frac{1}{2}}^{-} h_{N^{\prime}+\frac{1}{2}}^{+} h_{N^{\prime}-\frac{1}{2}}^{+} & =h_{N^{\prime}-\frac{1}{2}}^{+} r_{N^{\prime}-1}, & & \\
h_{i+\frac{3}{2}}^{-} h_{i+\frac{3}{2}}^{+} h_{i+\frac{1}{2}}^{+} & =h_{i+\frac{1}{2}}^{+} h_{i-\frac{1}{2}}^{+} h_{i-\frac{1}{2}}^{-} & & \text {for } i=\left[N^{\prime}+1, N-3\right] \cap I_{3} .
\end{aligned}
$$

and we require that the map

$$
H=h_{\frac{1}{2}}^{+}+h_{\frac{3}{2}}^{+}+\cdots+h_{N-\frac{1}{2}}^{+} \in \bigoplus_{i \in I_{1} \cup I_{2} \cup I_{3}} \operatorname{Hom}\left(V_{i}, V_{i+1}\right)
$$

has a type given by the partitions $\left\{\pi^{[a, b]} \mid 0 \leq a, b \leq N-1\right\}$. The linear map $H$ contains all the information of the maps $h_{i+\frac{1}{2}}^{+}$. For brevity, we make the following definition, packaging all the remaining linear maps into one.

Definition 4.11. Given a representation as above, we define the linear map

$$
\begin{aligned}
L:=r_{0}+r_{1}+\cdots+r_{N^{\prime}-1}+h_{N^{\prime}+\frac{1}{2}}^{-}+\cdots & +h_{N-\frac{3}{2}}^{-} \\
& \in \bigoplus_{i \in I_{1}} \operatorname{Hom}\left(V_{i}, V_{i}\right) \bigoplus_{i \in I_{2}} \operatorname{Hom}\left(V_{i+1}, V_{i}\right) .
\end{aligned}
$$

From now on, in order to compute the motivic class of $R\left(\left\{\pi^{[a, b]}\right\}\right)$ we will work with a choice of coordinates. Let

$$
v_{l}^{a, b}(k) \in V_{a}
$$

be such that $v_{l}^{a, b}(k)$ generates the $k$-th summand of $\mathbb{C}[x] /\left(x^{N(l-1)+|b-a|+1}\right)^{\oplus b_{l}^{a, b}}$ in the decomposition of Proposition 4.5. Then we have that $\mathcal{B}:=\left\{H^{p} v_{l}^{a, b}(k)\left|1 \leq k \leq b_{l}^{a, b}, 0 \leq a, b \leq N-1,0 \leq p \leq N(l-1)+\right| b-a \mid+1\right\}$ forms a basis of $V$. 
Definition 4.12. We define $H\left(\pi^{[a, b]}\right)$ to be the matrix representation of the map $H$ with respect to the basis $\mathcal{B}$. Also define

$$
\begin{aligned}
& F\left(\left\{\pi^{[a, b]}\right\}\right):=\left\{L \mid\left(L, H\left(\pi^{[a, b]}\right)\right) \in R\left(\left\{\pi^{[a, b]}\right\}\right)\right\}, \\
& N\left(\left\{\pi^{[a, b]}\right\}\right):=\left\{H \mid H \text { has type }\left\{\pi^{[a, b]}\right\}\right\} .
\end{aligned}
$$

Then $R\left(\left\{\pi^{[a, b]}\right\}\right)$ has a decomposition as a vector bundle:

Lemma 4.13. $R\left(\left\{\pi^{[a, b]}\right\}\right)$ has the structure of a vector bundle

$$
\begin{gathered}
F\left(\left\{\pi^{[a, b]}\right\}\right) \longrightarrow \\
\qquad\left(\left\{\pi^{[a, b]}\right\}\right) \\
\\
N\left(\left\{\pi^{[a, b]}\right\}\right)
\end{gathered}
$$

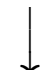

In particular, we have that

$$
\left[R\left(\left\{\pi^{[a, b]}\right\}\right)\right]=\left[F\left(\left\{\pi^{[a, b]}\right\}\right)\right] \cdot\left[N\left(\left\{\pi^{[a, b]}\right\}\right)\right]
$$

in the Grothendieck ring of varieties.

Proof. The projection map

$$
\begin{aligned}
p: R\left(\left\{\pi^{[a, b]}\right\}\right) & \rightarrow N\left(\left\{\pi^{[a, b]}\right\}\right), \\
(L, H) & \mapsto H
\end{aligned}
$$

defines the bundle structure with zero section $H \mapsto(0, H)$. The fiber is the linear space of all such $L$.

Here the base of the vector bundle is the space of all matrices of type $\left\{\pi^{[a, b]}\right\}$; these are all conjugate to $H\left(\pi^{[a, b]}\right)$, and therefore we have a torsor

$$
\begin{aligned}
\pi: G_{\alpha} & \rightarrow N\left(\pi^{[a, b]}\right), \\
P & \mapsto P H\left(\pi^{[a, b]}\right) P^{-1} .
\end{aligned}
$$

This is a torsor for the group $S^{\prime}\left(\left\{\pi^{[a, b]}\right\}\right):=\operatorname{Stab}_{G_{\alpha}}\left(H\left(\pi^{a, b}\right)\right)$. This group is given as the group of units in an algebra:

Definition 4.14. We identify $S^{\prime}\left(\left\{\pi^{[a, b]}\right\}\right)$ with the group of multiplicative units in the algebra

$$
S\left(\left\{\pi^{[a, b]}\right\}\right):=\left\{N \in \prod_{i=0}^{N-1} \operatorname{End}\left(\alpha_{i}\right) \mid N H\left(\pi^{[a, b]}\right)=H\left(\pi^{[a, b]}\right) N\right\} .
$$

Since $S^{\prime}\left(\left\{\pi^{[a, b]}\right\}\right)$ is the group of units of an algebra it is a special group [Chevalley et al. 1958], and so the above torsor splits in the Zariski topology. For completeness we include a short proof of this fact. 
Lemma 4.15. The group $S^{\prime}\left(\left\{\pi^{[a, b]}\right\}\right)$ is a special algebraic group.

Proof. The ring of units is defined by a single polynomial equation and is open in A. Consequently the units $S^{\prime}\left(\left\{\pi^{[a, b]}\right\}\right)$ act generically freely on the vector space $S\left(\left\{\pi^{[a, b]}\right\}\right)$, so by Proposition 3.13 of [Merkurjev 2013] the group has essential dimension zero. Then by Proposition 3.16 of [Merkurjev 2013] it is a special algebraic group.

The next lemma gives a formula of the motivic class of the group $S^{\prime}\left(\left\{\pi^{[a, b,]}\right\}\right)$, and via the splitting of the above torsor we deduce a formula for the class of $N\left(\left\{\pi^{[a, b]}\right\}\right)$. Before stating the lemma we create some notation:

Definition 4.16. We denote the dimensions of the linear spaces $F\left(\left\{\pi^{[a, b]}\right\}\right)$ and $S\left(\left\{\pi^{[a, b]}\right\}\right)$ by

$$
\begin{aligned}
& T\left(\left\{\pi^{[a, b]}\right\}\right):=\operatorname{dim} F\left(\left\{\pi^{[a, b]}\right\}\right), \\
& B\left(\left\{\pi^{[a, b]}\right\}\right):=\operatorname{dim} S\left(\left\{\pi^{[a, b]}\right\}\right) .
\end{aligned}
$$

Lemma 4.17. We have

$$
\left[S^{\prime}\left(\left\{\pi^{[a, b]}\right\}\right)\right]=\left[S\left(\left\{\pi^{[a, b]}\right\}\right)\right] \cdot \prod_{0 \leq a, b \leq N-1} \frac{1}{f\left(\pi^{[a, b]}\right)},
$$

where

$$
f\left(\pi^{[a, b]}\right):=\prod_{l \geq 1} \frac{\left[\operatorname{End}\left(b_{l}^{a, b}\right)\right]}{\left[\operatorname{GL}\left(b_{l}^{a, b}\right)\right]} .
$$

As a consequence,

$$
\left[R\left(\left\{\pi^{a, b}\right\}\right)\right]=\left[G_{\alpha}\right] \cdot \mathbb{\bigsqcup}^{T\left(\left\{\pi^{[a, b]}\right\}\right)-B\left(\left\{\pi^{[a, b]}\right\}\right)} \cdot \prod_{0 \leq a, b \leq N-1} f\left(\pi^{[a, b]}\right) .
$$

Proof. Let

$$
W_{l}^{a, b}:=\operatorname{span}_{\mathbb{C}}\left\{v_{l}^{a, b}(k) \mid 1 \leq k \leq b_{l}^{a, b}\right\}
$$

be the span of the basis elements $v_{l}^{a, b}(k)$ for $1 \leq k \leq b_{l}^{a, b}$. We have both inclusion and projection

$$
W_{l}^{a, b} \hookrightarrow V \rightarrow W_{l}^{a, b}
$$

This gives a map of algebras

$$
\begin{aligned}
\pi: S\left(\left\{\pi^{[a, b]}\right\}\right) & \rightarrow \prod_{a, b, l} \operatorname{End}\left(W_{l}^{a, b}\right), \\
N & \left.\mapsto \bigoplus_{a, b, l} N\right|_{W_{l}^{a, b}}
\end{aligned}
$$


This splits as a trivial vector bundle, whose rank is the dimension of the total space minus the dimension of the base. Since we have that the group $S^{\prime}\left(\left\{\pi^{[a, b]}\right\}\right)$ is the group of units in $S\left(\left\{\pi^{[a, b]}\right\}\right)$, we can identify $S^{\prime}\left(\left\{\pi^{[a, b]}\right\}\right)$ as the inverse image of the units on the right-hand side. This is a trivial vector bundle of rank equal to $\operatorname{dim} S\left(\left\{\pi^{[a, b]}\right\}\right)-\operatorname{dim} \prod_{a, b, l} \operatorname{End}\left(W_{l}^{a, b}\right)$. We have an isomorphism of varieties

$$
S^{\prime}\left(\left\{\pi^{[a, b]}\right\}\right) \equiv \frac{S\left(\left\{\pi^{[a, b]}\right\}\right)}{\prod_{a, b, l} \operatorname{End}\left(W_{l}^{a, b}\right)} \times \prod_{a, b, l} \operatorname{GL}\left(W_{l}^{a, b}\right),
$$

so motivically we have

$$
\left[S^{\prime}\left(\left\{\pi^{[a, b]}\right\}\right)\right]=\left[S\left(\left\{\pi^{[a, b]}\right\}\right)\right] \cdot \prod_{0 \leq a, b \leq N-1} \frac{1}{f\left(\pi^{[a, b]}\right)} .
$$

In Lemma 4.13 we saw that

$$
\left[R\left(\left\{\pi^{[a, b]}\right\}\right)\right]=\left[F\left(\left\{\pi^{[a, b]}\right\}\right)\right] \cdot\left[N\left(\left\{\pi^{[a, b]}\right\}\right)\right] .
$$

Now we know that $N\left(\left\{\pi^{[a, b]}\right\}\right)$ is a torsor for the group $S^{\prime}\left(\left\{\pi^{[a, b]}\right\}\right)$. We have just computed the motive of this group. So we can deduce that

$$
\begin{aligned}
{\left[R\left(\left\{\pi^{[a, b]}\right\}\right)\right] } & =\left[F\left(\left\{\pi^{[a, b]}\right\}\right)\right] \cdot \frac{\left[G_{\alpha}\right]}{\left[S^{\prime}\left(\left\{\pi^{[a, b]}\right\}\right)\right]} \\
& =\left[F\left(\left\{\pi^{[a, b]}\right\}\right)\right] \cdot \frac{\left[G_{\alpha}\right]}{\left[S\left(\left\{\pi^{[a, b]}\right\}\right)\right]} \cdot \prod_{0 \leq a, b \leq N-1} f\left(\pi^{[a, b]}\right) \\
& =\left[G_{\alpha}\right] \cdot \mathbb{L}^{T\left(\left\{\pi^{[a, b]}\right\}\right)-B\left(\left\{\pi^{[a, b]}\right\}\right)} \cdot \prod_{0 \leq a, b \leq N-1} f\left(\pi^{[a, b]}\right) .
\end{aligned}
$$

The next proposition computes the difference $T\left(\left\{\pi^{[a, b]}\right\}\right)-B\left(\left\{\pi^{[a, b]}\right\}\right)$. Its proof is found in the Appendix:

Proposition 4.18. We have that $T\left(\left\{\pi^{[a, b]}\right\}\right)-B\left(\left\{\pi^{[a, b]}\right\}\right)$ is equal to

$$
\begin{aligned}
-\frac{1}{2} \sum_{i \in I_{2}}\left(\sum_{b \neq i} l\left(\pi^{[i+1, b]}\right)-\sum_{c \neq i+1} l\left(\pi^{[c, i]}\right)\right)^{2} & -\frac{1}{2} \sum_{\substack{a \in I_{3} \\
b \notin I_{2}}} \sum_{i \geq 1}\left(b_{i}^{a, b}\right)^{2}-\frac{1}{2} \sum_{\substack{a \notin I_{3} \\
b \in I_{2}}} \sum_{i \geq 1}\left(b_{i}^{a, b}\right)^{2} .
\end{aligned}
$$

Proof. The proof is a linear algebra calculation. See the Appendix.

As a corollary, we deduce the formula for $N^{\sigma}(y)$ : 
Proposition 4.19. Let

$$
\begin{aligned}
S & =\left\{[a, b] \mid a \in I_{3}, b \notin I_{2} \text { or } a \notin I_{3}, b \in I_{2}\right\}, \\
y_{[a, b]} & =y_{a} \cdot y_{a+1} \cdots y_{b}, \\
y^{\prime} & =y_{0} \cdot y_{1} \cdots y_{N-1},
\end{aligned}
$$

then we have

$$
N^{\sigma}(y)=\operatorname{Exp}\left(\frac{\mathbb{L}}{\mathbb{L}-1} \frac{1}{1-y^{\prime}}\left(\sum_{[a, b] \notin S} y_{[a, b]}-\mathbb{L}^{-\frac{1}{2}} \sum_{[a, b] \in S} y_{[a, b]}\right)\right) .
$$

Proof. Recall our initial definition of $N^{\sigma}(y)$ :

$$
N^{\sigma}(y)=\sum_{\alpha \in \mathbb{N} Q_{0}}\left(-\mathbb{L}^{\frac{1}{2}}\right)^{\chi(\alpha, \alpha)+2 d_{C}(\alpha)} \frac{\left[R^{N}(\alpha)\right]}{\left[G_{\alpha}\right]} y^{\alpha} .
$$

In Proposition 4.5 we saw that it was possible to stratify each of the varieties $R^{N}(\alpha)$ by the type $\left\{\pi^{[a, b]}\right\}$ of the cycle $H$. This gives

$$
N^{\sigma}(y)=\sum_{\alpha \in \mathbb{N} Q_{0}}\left(-\mathbb{L}^{\frac{1}{2}}\right)^{\chi(\alpha, \alpha)+2 d_{C}(\alpha)}\left[G_{\alpha}\right]^{-1}\left(\sum_{\left\{\pi^{[a, b]}\right\} \vdash \alpha}\left[R\left(\left\{\pi^{[a, b]}\right\}\right)\right]\right) y^{\alpha} .
$$

The motivic class of $R\left(\left\{\pi^{[a, b]}\right\}\right)$ was computed in Lemma 4.17. Now substituting this class into the above formula gives

$$
\begin{aligned}
\sum_{\alpha \in \mathbb{N} Q_{0}}\left(-\mathbb{L}^{\frac{1}{2}}\right)^{\chi(\alpha, \alpha)+2 d_{C}(\alpha)} & \cdot\left(\sum_{\left\{\pi^{[a, b]}\right\} \vdash \alpha} \mathbb{L}^{T\left(\left\{\pi^{[a, b]}\right\}\right)-B\left(\left\{\pi^{[a, b]}\right\}\right)} \cdot \prod_{0 \leq a, b \leq N-1} f\left(\pi^{[a, b]}\right)\right) y^{\alpha} .
\end{aligned}
$$

Lemma 4.8 showed how the dimension vector depended on the partitions: we had

$$
\alpha_{i}=\sum_{0 \leq a, b \leq N-1}\left|\pi^{[a, b]}\right|-\sum_{[a, b] \ngtr i} l\left(\pi^{[a, b]}\right),
$$

and an immediate corollary was that

$$
\chi(\alpha, \alpha)+2 d_{C}(\alpha)=\sum_{i \in I_{2}}\left(\sum_{b \neq i} l\left(\pi^{[i+1, b]}\right)-\sum_{c \neq i+1} l\left(\pi^{[c, i]}\right)\right)^{2} .
$$


Combining this with the formula for the difference $T\left(\left\{\pi^{[a, b]}\right\}\right)-B\left(\left\{\pi^{[a, b]}\right\}\right)$ (Proposition 4.18) gives

$$
\begin{aligned}
N^{\sigma}(y)=\sum_{\left\{\pi^{[a, b]\}}\right.}\left(\prod_{[a, b] \notin S} f\left(\pi^{[a, b]}\right)\right) & \cdot\left(\prod_{[a, b] \in S} f\left(\pi^{[a, b]}\right) \prod_{l \geq 1}\left(-\mathbb{L}^{\frac{1}{2}}\right)^{-\left(b_{l}^{a, b}\right)^{2}}\right) \\
& \cdot \prod_{i=0}^{N-1} y_{i}^{\sum_{0 \leq a, b \leq N-1}\left|\pi^{[a, b]}\right|-\sum_{[a, b] \ngtr i} l\left(\pi^{[a, b]}\right)} .
\end{aligned}
$$

To simplify notation, set

$$
g(\pi):=f(\pi) \cdot \prod_{l \geq 1}\left(-\mathbb{1}^{\frac{1}{2}}\right)^{-b_{l}^{2}} \quad \text { for } \pi=\left(1^{b_{1}} 2^{b_{2}} 3^{b_{3}} \cdots\right) ;
$$

then rearranging the products and summations gives

$$
\begin{aligned}
& N^{\sigma}(y)= \prod_{[a, b] \notin S} \sum_{\pi^{[a, b]}} f\left(\pi^{[a, b]}\right) \cdot y^{\prime\left|\pi^{[a, b]}\right|-l\left(\pi^{[a, b]}\right)} \cdot y_{[a, b]}^{l\left(\pi^{[a, b]}\right)} \\
& \cdot \prod_{[a, b] \in S} \sum_{\pi^{[a, b]}} g\left(\pi^{[a, b]}\right) \cdot y^{|| \pi^{[a, b]} \mid-l\left(\pi^{[a, b]}\right)} \cdot y_{[a, b]}^{l\left(\pi^{[a, b]}\right)} .
\end{aligned}
$$

Both of these series are know to have product expansions [Macdonald 1995]

$$
\begin{aligned}
& f(t, a)=\sum_{\pi} f(\pi) a^{l(\pi)} t^{|\pi|-l(\pi)}=\operatorname{Exp}\left(\frac{1}{1-\mathbb{L}^{-1}} \cdot \frac{a}{1-t}\right), \\
& g(t, a)=\sum_{\pi} g(\pi) a^{l(\pi)} t^{|\pi|-l(\pi)}=\operatorname{Exp}\left(\frac{\left(-\mathbb{L}^{\frac{1}{2}}\right)^{-1}}{1-\mathbb{L}^{-1}} \cdot \frac{a}{1-t}\right) .
\end{aligned}
$$

Now $N^{\sigma}$ is a product of such series, and multiplying together the corresponding exponential generating series gives the desired result

$$
N^{\sigma}(y)=\operatorname{Exp}\left(\frac{\mathbb{L}}{\mathbb{L}-1} \frac{1}{1-y^{\prime}}\left(\sum_{[a, b] \notin S} y_{[a, b]}-\mathbb{L}^{-\frac{1}{2}} \sum_{[a, b] \in S} y_{[a, b]}\right)\right) .
$$

Now we have computed $I^{\sigma}$ and $N^{\sigma}$, and so by Lemma 4.3

$$
A_{U}^{\sigma}(y)=\operatorname{Exp}\left(\mathbb{L} \frac{y^{\prime}}{1-y^{\prime}}+\frac{\mathbb{L}}{\mathbb{L}-1} \frac{1}{1-y^{\prime}}\left(\sum_{[a, b] \notin S} y_{[a, b]}-\mathbb{L}^{-\frac{1}{2}} \sum_{[a, b] \in S} y_{[a, b]}\right)\right) .
$$

Or, reformulating this as a product over the set of roots, we get

$$
\operatorname{Exp}\left(\frac{1}{1-\mathbb{L}^{-1}}\left((\mathbb{L}+N-1) \sum_{\alpha \in \Delta_{\sigma,+}^{\mathrm{im}}} y^{\alpha}+\sum_{\substack{\alpha \in \Delta_{\sigma,+}^{\mathrm{re}} \\ \sum_{I_{2} \cup I_{3}} \alpha_{i} \text { even }}} y^{\alpha-\mathbb{L}^{-\frac{1}{2}}} \sum_{\substack{\alpha \in \Delta_{\sigma,+}^{\mathrm{re}} \\ \sum_{I_{2} \cup I_{3}} \alpha_{i} \text { odd }}} y^{\alpha}\right)\right),
$$


thus proving Theorem 0.1 for the special case of the partition $\sigma$ :

$$
A_{U}^{\sigma}(y)=\prod_{\alpha \in \Delta_{\sigma,+}} A^{\alpha}(y)
$$

\section{The universal DT series: general case}

In this section we will prove Theorem 0.1 for any partition $\sigma$.

5A. Mutation and the root system. Recall that the simple reflection provides a bijection between $\Delta_{\sigma,+} \backslash\left\{\alpha_{k}\right\}$ and $\Delta_{\sigma^{\prime},+} \backslash\left\{\alpha_{k}^{\prime}\right\}$ (see Section 2C). The simple root $\alpha_{k}$ maps to $-\alpha_{k}^{\prime}$.

For $\alpha \in \Delta_{+}^{\mathrm{re}}$, let $x_{\alpha}$ be a simple module of $\operatorname{dim} \alpha$. By [Nagao 2012, Proposition 2.14], $\sum_{i \notin \widehat{I}_{r}} \alpha_{i}$ is odd (resp. even) if and only if $\operatorname{ext}^{1}(x, x)=0$ (resp. $\left.=1\right)$. In particular, the parity of $\sum_{i \notin \hat{I}_{r}} \alpha_{i}$ is preserved by the simple reflection.

\section{B. Wall-crossing formula.}

Theorem 5.1 [Nagao 2011c, Theorem 4.9].

$$
A_{U}^{\sigma^{\prime}}(\boldsymbol{y})=\frac{A_{U}^{\sigma}(\boldsymbol{y})}{\mathbb{E}\left(y_{k}\right)} \times \mathbb{E}\left(y_{k}^{-1}\right) .
$$

Proof. Step 1: By the observation in Section 2C, we have the factorization

$$
A_{U}^{\sigma}=\mathbb{E}\left(y_{k}\right) \times A_{k}^{\sigma},
$$

where

$$
\mathbb{E}(y):=\sum_{n \geq 0} \frac{[\mathrm{pf}]}{\left[\mathrm{GL}_{n}\right]_{\mathrm{vir}}} \cdot y^{n}, \quad y_{k}:=y_{\alpha_{k}}
$$

and $A_{k}^{\sigma}$ is the generating series of virtual motives of moduli stacks of objects in $\left(\bmod J_{\sigma}\right)_{k}$. We also have

$$
A_{U}^{\sigma^{\prime}}=A^{\sigma^{\prime}, k} \times \mathbb{E}\left(y_{k}^{-1}\right)
$$

where $A^{\sigma^{\prime}, k}$ is the generating series of virtual motives of moduli stacks of objects in $\left(\bmod J_{\sigma^{\prime}}\right)^{k}$.

Step 2: By Proposition 2.4, we have $A_{k}^{\sigma}=A^{\sigma^{\prime}, k}$ (see [Nagao 2011c, Theorem 4.7]).

Now Theorem 0.1 follows for any $\sigma$ from the result in Section 4 combined with Theorem 5.1 and the remark in Section 5A. 
5C. Factorization of the universal series. We will say that a stability parameter $\zeta$ is generic, if for any stable $J_{\sigma}$-module $V$, we have $\zeta \cdot \underline{\operatorname{dim}} V \neq 0$. For generic stability parameter $\zeta$, let $\mathfrak{M}_{\zeta}^{+}\left(J_{\sigma}, \alpha\right)$ (resp. $\left.\mathfrak{M}_{\zeta}^{-}\left(J_{\sigma}, \alpha\right)\right)$ denote the moduli stacks of $J_{\sigma}$-modules $V$ such that $\underline{\operatorname{dim}} V=\alpha$ and such that all the HN factors $F$ of $V$ with respect to the stability parameter $\zeta$ satisfy $\zeta \cdot \underline{\operatorname{dim}} F>0$ (resp. $<0$ ). Let $\left[\mathfrak{M}_{\zeta}^{ \pm}\left(J_{\sigma}, \alpha\right)\right]_{\text {vir }}$ denote the virtual motive of the moduli stack defined in the same way as (3-2). We put

$$
A_{\zeta}^{ \pm}(y)=\sum_{\alpha \in \mathbb{N}^{I}}\left[\mathfrak{M}_{\zeta}^{ \pm}(J, \alpha)\right]_{\mathrm{vir}} \cdot y^{\alpha} .
$$

Lemma 5.2 [Morrison et al. 2012, Lemma 2.6]. The generating series $A_{\zeta}^{ \pm}$are given by

$$
A_{\zeta}^{ \pm}(y)=\prod_{\substack{\alpha \in \Delta_{\sigma,+} \\ \pm \zeta \cdot \alpha<0}} A^{\alpha}(y)
$$

\section{Motivic DT with framing and DT/PT series}

6A. Motivic DT invariants with framing. We denote by $\widetilde{Q}_{\sigma}$ the new quiver obtained from $Q_{\sigma}$ by adding a new vertex $\infty$ and a single new arrow $\infty \rightarrow 0$. Let $\tilde{J}_{\sigma}=J_{\widetilde{Q}_{\sigma}, w_{\sigma}}$ be the Jacobian algebra corresponding to the quiver with potential $\left(\widetilde{Q}_{\sigma}, w_{\sigma}\right)$, where we view $w_{\sigma}$ as a potential for $\widetilde{Q}_{\sigma}$ in the obvious way.

Let $\zeta \in \mathbb{R}^{\widehat{I}}$ be a vector, which we will refer to as the stability parameter. A $\widetilde{J}_{\sigma^{-}}$ representation $\tilde{V}$ with $\operatorname{dim} \widetilde{V}_{\infty}=1$ is said to be $\zeta$-(semi)stable, if it is (semi)stable with respect to $\left(\zeta, \zeta_{\infty}\right) \in \mathbb{R}^{\hat{I} \sqcup\{\infty\}}$ (see Definition 3.3), where $\zeta_{\infty}=-\zeta \cdot \underline{\operatorname{dim}} V$. As in Section $3 \mathrm{~B}$, a stability parameter $\zeta \in \mathbb{R}^{Q_{0}}$ is said to be generic if for any stable $J$-module $V$ we have $\zeta \cdot \underline{\operatorname{dim}} V \neq 0$.

For a stability parameter $\zeta \in \mathbb{R}^{Q_{0}}$ and a dimension vector $\alpha \in\left(\mathbb{Z}_{\geq 0}\right)^{\hat{I}}$, let $\mathfrak{M}_{\zeta}\left(\widetilde{J}_{\sigma}, \alpha\right)$ denote the moduli stack of $\zeta$-semistable $\widetilde{J}_{\sigma}$-representations with dimension vector $(\alpha, 1)$. As in the introduction, we define the generating function

$$
Z_{\zeta}\left(y_{0}, \ldots, y_{N-1}\right)=Z_{\zeta}(y):=\sum_{\alpha \in\left(\mathbb{Z}_{\geq 0}\right)^{\widehat{I}}}\left[\mathfrak{M}_{\zeta}\left(\widetilde{J}_{\sigma}, \alpha\right)\right]_{\text {vir }} \cdot y^{\alpha} .
$$

Theorem 6.1 [Morrison et al. 2012, Proposition 4.6]. For a generic stability parameter $\zeta$, we have

$$
Z_{\zeta}(y)=\frac{A_{\zeta}^{-}\left(-\llbracket^{\frac{1}{2}} y_{0}, y_{1}, \ldots, y_{N-1}\right)}{A_{\zeta}^{-}\left(-\mathbb{L}^{-\frac{1}{2}} y_{0}, y_{1}, \ldots, y_{N-1}\right)},
$$

where $A_{\zeta}^{-}$was defined in Section $5 C$.

Combined with Lemma 5.2, we get the formula in Corollary 0.2. 
Remark 6.2. If we cross the wall $W_{\alpha}$, we get (or lose) a factor $Z_{\alpha}(y)$ in the generating function. This is compatible with the result in [Nagao 2011b].

6B. Chambers in the moduli spaces. For a root $\alpha \in \Lambda$, let $W_{\alpha}$ denote the hyperplane in the space $\mathbb{R}^{\widehat{I}}$ of stability parameters which is orthogonal to $\alpha$. We put

$$
W=W_{\delta} \cup \bigcup_{\alpha \in \Delta_{\sigma,+}^{\mathrm{re}}} W_{\alpha}
$$

A connected component of the complement of $W$ in $\mathbb{R}^{\hat{I}}$ is called a chamber.

Theorem 6.3 [Nagao 2012, Proposition 2.10; Nagao and Nakajima 2011, Propositions 3.10, 3.11]. The set of generic parameters in $\mathbb{R}^{\widehat{I}}$ is the compliment of $W$.

(i) For $\zeta$ with $\zeta_{i}<0$ for all $i$, the moduli spaces $\mathfrak{M}_{\zeta}(\widetilde{J}, \alpha)$ are the NCDT moduli spaces, the moduli spaces of cyclic $J$-modules from [Szendrối 2008].

(ii) For $\zeta$ in the same chamber as $(1-N+\varepsilon, 1,1, \ldots, 1)(0<\varepsilon \ll 1)$, the moduli spaces $\mathfrak{M}_{\zeta}(\widetilde{J}, \alpha)$ are the DT moduli spaces of $Y_{\sigma}$ from [Maulik et al. 2006], the moduli spaces of subschemes on $Y_{\sigma}$ with support in dimension at most 1.

(iii) For $\zeta$ in the same chamber as $(1-N-\varepsilon, 1,1, \ldots, 1)(0<\varepsilon \ll 1)$, the moduli spaces $\mathfrak{M}_{\zeta}(\widetilde{J}, \alpha)$ are the PT moduli spaces of $Y_{\sigma}$ introduced in [Pandharipande and Thomas 2009]; these are moduli spaces of stable rank-1 coherent systems.

Remark 6.4. In the above statements $\varepsilon$ depends on the dimension vector $(\alpha, 1)$.

6C. Motivic PT and DT invariants. Let

$$
\zeta_{\mathrm{DT}}=(1-N-\varepsilon, 1,1, \ldots, 1), \quad \zeta_{\mathrm{PT}}=(1-N+\varepsilon, 1,1, \ldots, 1) \quad(0<\varepsilon \ll 1)
$$

be stability parameters corresponding to DT and PT moduli spaces. Then we have

$$
\begin{aligned}
& \left\{\alpha \in \Delta_{\sigma,+} \mid \zeta_{\mathrm{DT}} \cdot \alpha<0\right\}=\Delta_{+}^{\mathrm{re},+}, \\
& \left\{\alpha \in \Delta_{\sigma,+} \mid \zeta_{\mathrm{PT}} \cdot \alpha<0\right\}=\Delta_{+}^{\mathrm{re},+} \sqcup \Delta_{+}^{\mathrm{im}} .
\end{aligned}
$$

As we mentioned in the introduction, the variable change induced by the derived equivalence is given by

$$
s:=y_{0} \cdot y_{1} \cdots y_{N-1}, \quad T_{i}=y_{i} .
$$

Here $s$ is the variable for the homology class of a point and $T_{i}$ is the variable for the homology class of $C_{i}$. Then we get the formulae in Corollary 0.3. 
6D. Connection with the refined topological vertex. As in [Nagao 2011a], we can apply the vertex operator method [Okounkov et al. 2006] to get a product expansion of the refined topological vertex for $\mathcal{Y}_{\sigma}$. Then we see that the PT generating function can be described by the refined topological vertices normalized by the refined MacMahon functions. ${ }^{5}$

\section{Appendix}

Throughout this appendix we will work with a fixed choice of basis $\mathcal{B}$. In Section 4B we chose a basis

$\mathcal{B}=\left\{H^{p} v_{l}^{a, b}(k)\left|1 \leq k \leq b_{l}^{a, b}, 0 \leq a, b \leq N-1,0 \leq p \leq N(l-1)+\right| b-a \mid+1\right\}$

and defined linear spaces

$$
\begin{aligned}
& F\left(\left\{\pi^{[a, b]}\right\}\right) \\
& =\left\{L \in \bigoplus_{i \in I_{1}} \operatorname{Hom}\left(V_{i}, V_{i}\right) \oplus \bigoplus_{i \in I_{2}} \operatorname{Hom}\left(V_{i+1}, V_{i}\right) \mid\left(L, H\left(\pi^{[a, b]}\right)\right) \in R\left(\left\{\pi^{[a, b]}\right\}\right)\right\}, \\
& S\left(\left\{\pi^{[a, b]}\right\}\right)=\left\{N \in \bigoplus_{i \in I_{1} \cup I_{2} \cup I_{3}} \operatorname{Hom}\left(V_{i}, V_{i}\right) \mid\left[N, H\left(\pi^{[a, b]}\right)\right]=0\right\},
\end{aligned}
$$

with dimensions $T\left(\left\{\pi^{[a, b]}\right\}\right)=\operatorname{dim} F\left(\left\{\pi^{[a, b]}\right\}\right)$ and $B\left(\left\{\pi^{[a, b]}\right\}\right)=\operatorname{dim} S\left(\left\{\pi^{[a, b]}\right\}\right)$. The goal of the appendix is to prove Proposition 4.18, that is, to show that the difference $T\left(\left\{\pi^{[a, b]}\right\}\right)-B\left(\left\{\pi^{[a, b]}\right\}\right)$ is equal to

$$
\begin{aligned}
-\frac{1}{2} \sum_{i \in I_{2}}\left(\sum_{b \neq i} l\left(\pi^{[i+1, b]}\right)-\right. & \left.\sum_{c \neq i+1} l\left(\pi^{[c, i]}\right)\right)^{2} \\
& \quad-\frac{1}{2} \sum_{a \in I_{3}, b \notin I_{2}} \sum_{i \geq 1}\left(b_{i}^{a, b}\right)^{2}-\frac{1}{2} \sum_{a \notin I_{3}, b \in I_{2}} \sum_{i \geq 1}\left(b_{i}^{a, b}\right)^{2} .
\end{aligned}
$$

For some early examples it becomes clear that the dimensions of $F\left(\left\{\pi^{[a, b]}\right\}\right)$ and $S\left(\left\{\pi^{[a, b]}\right\}\right)$ are determined by solving a set of linearly independent equations. We will see that these dimensions are quadratic polynomials in the number of parts $b_{l}^{a, b}$ of the partitions $\left\{\pi^{[a, b]}\right\}$. An initial means of simplifying the calculation is to break the spaces $F\left(\left\{\pi^{[a, b]}\right\}\right)$ and $S\left(\left\{\pi^{[a, b]}\right\}\right)$ down into simpler spaces. One easy observation is that not only are the spaces $F\left(\left\{\pi^{[a, b]}\right\}\right)$ and $S\left(\left\{\pi^{[a, b]}\right\}\right)$ linear, but they come with a natural vector space structure, the origin corresponding

${ }^{5}$ Unfortunately, the DT generating function does not coincide with the refined topological vertex. See [Morrison et al. 2012, §4.3] for details. 
to the zero matrix in both cases. This means that we have decompositions as vector spaces

$$
\begin{aligned}
& F\left(\left\{\pi^{[a, b]}\right\}\right)=\bigoplus_{0 \leq a, b, c, d \leq N-1} F\left(\pi^{[a, b]}, \pi^{[c, d]}\right), \\
& S\left(\left\{\pi^{[a, b]}\right\}\right)=\bigoplus_{0 \leq a, b, c, d \leq N-1} S\left(\pi^{[a, b]}, \pi^{[c, d]}\right)
\end{aligned}
$$

whose summands are given by the following definition:

Definition A.5. We define

$$
\begin{aligned}
& F\left(\pi^{[a, b]}, \pi^{[c, d]}\right)=F\left(\left\{\pi^{[a, b]}\right\}\right) \cap \bigoplus_{i \in I_{1} \cup I_{2}} \operatorname{Hom}\left(V^{a, b}, V^{c, d}\right), \\
& S\left(\pi^{[a, b]}, \pi^{[c, d]}\right)=S\left(\pi^{[a, b]}, \pi^{[c, d]}\right) \cap \bigoplus_{i \in I_{1} \cup I_{2} \cup I_{3}} \operatorname{Hom}\left(V^{a, b}, V^{c, d}\right) .
\end{aligned}
$$

These subspaces are essentially given by the block matrices for the decomposition $V=\bigoplus_{0 \leq a, b \leq N-1} V^{a, b}$.

Definition A.6. We define

$$
\begin{aligned}
& T\left(\pi^{[a, b]}, \pi^{[c, d]}\right)=\operatorname{dim} F\left(\pi^{[a, b]}, \pi^{[c, d]}\right) \\
& B\left(\pi^{[a, b]}, \pi^{[c, d]}\right)=\operatorname{dim} S\left(\pi^{[a, b]}, \pi^{[c, d]}\right) .
\end{aligned}
$$

Both $T\left(\pi^{[a, b]}, \pi^{[c, d]}\right)$ and $B\left(\pi^{[a, b]}, \pi^{[c, d]}\right)$ can be written as quadratic expressions in the number of parts of $\pi^{[a, b]}$ and $\pi^{[c, d]}$. To do so, we introduce a quadratic form on the space of all partitions and a combinatorial operation that removes a box from each column of the partition.

Definition A.7. We define

$$
\begin{aligned}
M: \mathcal{P} \otimes \mathcal{P} & \rightarrow \mathbb{Z}_{\geq 0}, \\
\left(1^{b_{1}} 2^{b_{2}} 3^{b_{3}} \cdots\right) \otimes\left(1^{c_{1}} 2^{c_{2}} 3^{c_{3}} \cdots\right) & \mapsto \sum_{i \geq 1}\left(\sum_{j \geq i} b_{j}\right)\left(\sum_{j \geq i} c_{j}\right), \\
: \mathcal{P} & \rightarrow \mathcal{P}, \\
\pi=\left(1^{b_{1}} 2^{b_{2}} 3^{b_{3}} \cdots\right) & \mapsto \pi^{\prime}=\left(1^{b_{2}} 2^{b_{3}} 3^{b_{4}} \cdots\right) .
\end{aligned}
$$

Let us begin with the easier case. We compute dimensions $B\left(\pi^{[a, b]}, \pi^{[c, d]}\right)$ of the spaces $S\left(\pi^{[a, b]}, \pi^{[c, d]}\right)$. 
Lemma A.8. Let $N \in S\left(\pi^{[a, b]}, \pi^{[c, d]}\right)$; then the matrix $N$ is uniquely determined by its value on the vectors $v_{l}^{a, b}(k)$. Moreover, the only restriction on the image of such a vector is that it lie in the linear subspace

$$
N\left(v_{l}^{a, b}\right) \in V_{a} \cap V^{c, d} \cap V^{N \cdot(l-1)+|b-a|+1} .
$$

Proof. To define the linear map $N$ on the space $V^{a, b}$, it suffices to define its value at each of the basis vectors

$$
\left\{H^{r} v_{l}^{a, b}(k)|0 \leq r \leq N \cdot(l-1)+| b-a \mid, 1 \leq k \leq b_{l}^{a, b}\right\} .
$$

However, for $N \in S\left(\pi^{[a, b]}, \pi^{[c, d]}\right)$, we have

$$
N\left(H^{r} v_{l}^{a, b}(k)\right)=H^{r}\left(N v_{l}^{a, b}(k)\right)
$$

therefore the value of $N$ at each $H^{r} v_{l}^{a, b}(k)$ is determined by $N v_{l}^{a, b}(k)$. This proves the first part of the lemma. Now we know that the matrix $N$ maps the vector space at the $a$-th vertex to itself: $V_{a} \rightarrow V_{a}$; also, since $N \in S\left(\pi^{[a, b]}, \pi^{[c, d]}\right)$, we insist that its image be in $V^{c, d}$. The only additional condition on the image of the vector $v_{l}^{a, b}(k)$ is

$$
H^{N \cdot(l-1)+|b-a|+1}\left(N v_{l}^{a, b}(k)\right)=N\left(H^{N \cdot(l-1)+|b-a|+1} v_{l}^{a, b}(k)\right)=0 .
$$

Combining these three conditions above, we have

$$
N\left(v_{l}^{a, b}(k)\right) \in V_{a} \cap V^{c, d} \cap V^{N \cdot(l-1)+|b-a|+1} .
$$

Corollary A.9. We have

$$
B\left(\pi^{[a, b]}, \pi^{[c, d]}\right)= \begin{cases}M\left(\pi^{[a, b]}, \pi^{[c, d]}\right) & \text { if } a \in[c, d] \text { and }|d-a| \leq|b-a|, \\ M\left(\left(\pi^{[a, b]}\right)^{\prime}, \pi^{[c, d]}\right) & \text { if } a \in[c, d] \text { and }|d-a|>|b-a|, \\ M\left(\pi^{[a, b]},\left(\pi^{[c, d]}\right)^{\prime}\right) & \text { if } a \notin[c, d] \text { and }|d-a| \leq|b-a|, \\ M\left(\left(\pi^{[a, b]}\right)^{\prime},\left(\pi^{[c, d]}\right)^{\prime}\right) & \text { if } a \notin[c, d] \text { and }|d-a|>|b-a| .\end{cases}
$$

Proof. Let $N \in S\left(\pi^{[a, b]}, \pi^{[c, d]}\right)$. Each vector $v_{l}^{a, b}(k)$ with $1 \leq k \leq b_{l}^{a, b}$ can take any value in the vector space $V_{a} \cap V^{c, d} \cap V^{N \cdot(l-1)+|b-a|+1}$, and so the dimension of $S\left(\pi^{a, b}, \pi^{[c, d]}\right)$ is given by

$$
B\left(\pi^{[a, b]}, \pi^{[c, d]}\right)=\sum_{l \geq 0} b_{l}^{a, b} \cdot \operatorname{dim}\left(V_{a} \cap V^{c, d} \cap V^{N \cdot(l-1)+|b-a|+1}\right) .
$$


Counting the number of basis vectors of $V^{c, d}$ that lie in $V_{a}$, we see there are four possibilities for $\operatorname{dim}\left(V_{a} \cap V^{c, d} \cap V^{N \cdot(l-1)+|b-a|+1}\right)$ :

$$
\begin{array}{ll}
\sum_{i=1}^{l} i b_{i}^{c, d}+l \sum_{i \geq l} b_{i}^{c, d} & \text { if } a \in[c, d] \text { and }|d-a| \leq|b-a|, \\
\sum_{i=1}^{l-1} i b_{i}^{c, d}+(l-1) \sum_{i \geq l} b_{i}^{c, d} & \text { if } a \in[c, d] \text { and }|d-a|>|b-a|, \\
\sum_{i=1}^{l} i b_{i+1}^{c, d}+l \sum_{i \geq l} b_{i+1}^{c, d} & \text { if } a \notin[c, d] \text { and }|d-a| \leq|b-a|, \\
\sum_{i=1}^{l-1} i b_{i+1}^{c, d}+(l-1) \sum_{i \geq l} b_{i+1}^{c, d} & \text { if } a \notin[c, d] \text { and }|d-a|>|b-a| .
\end{array}
$$

Consider the first case $a \in[c, d]$ and $|d-a| \leq|b-a|$; then

$$
\begin{aligned}
B\left(\pi^{[a, b]}, \pi^{[c, d]}\right) & =\sum_{l \geq 1} b_{l}^{a, b} \cdot\left(\sum_{i=1}^{l} i b_{i}^{c, d}+l \sum_{i \geq l} b_{i}^{c, d}\right) \\
& =\sum_{i \geq 1}\left(\sum_{l \geq i} b_{l}^{a, b}\right) \cdot\left(\sum_{l \geq i} b_{l}^{c, d}\right) \\
& =M\left(\pi^{[a, b]}, \pi^{[c, d]}\right) .
\end{aligned}
$$

The other three cases are identical. The relabeling of the partitions in these cases is encoded by the operation $\pi \mapsto \pi^{\prime}$.

Now we turn to computing the dimensions $T\left(\pi^{[a, b]}, \pi^{[c, d]}\right)$ of the spaces $F\left(\pi^{[a, b]}, \pi^{[c, d]}\right)$. This will be more intricate.

Lemma A.10. Suppose $a \in I_{1} \cup I_{3}$ and $L \in F\left(\pi^{[a, b]}, \pi^{[c, d]}\right)$; then the map $L$ is uniquely determined by its value on the vectors $v_{l}^{a, b}(k)$. Moreover the only restriction on the image of such a vector is that it lie in a linear subspace:

$$
L v_{l}^{a, b}(k) \in\left\{\begin{array}{cl}
V_{a} \cap V^{N \cdot(l-1)+|b-a|+1} \cap V^{c, d} & \text { if } a \in I_{1} \text { and } b \notin I_{2}, \\
V_{a} \cap V^{N \cdot(l-1)+|b-a|} \cap V^{c, d} & \text { if } a \in I_{1} \text { and } b \in I_{2}, \\
V_{a-1} \cap V^{N \cdot(l-1)+|b-a|+2} \cap V^{c, d} & \text { if } a \in I_{3} \text { and } b \notin I_{2}, \\
V_{a-1} \cap V^{N \cdot(l-1)+|b-a|+1} \cap V^{c, d} & \text { if } a \in I_{3} \text { and } b \in I_{2} .
\end{array}\right.
$$

Proof. To define the linear map $L$ on the space $V^{a, b}$, it suffices to define its value at each of the basis vectors

$$
\left\{H^{r} v_{l}^{a, b}(k)|0 \leq r \leq N \cdot(l-1)+| b-a \mid, 1 \leq k \leq b_{l}^{a, b}\right\} .
$$


However, for $L \in F\left(\pi^{[a, b]}, \pi^{[c, d]}\right)$, we know that the pair $\left(L, H\left(\pi^{[a, b]}\right)\right) \in$ $R\left(\left\{\pi^{[a, b]}\right\}\right)$ satisfies the relations coming from the superpotential:

$$
\begin{aligned}
r_{i} h_{i-\frac{1}{2}}^{+} & =h_{i-\frac{1}{2}}^{+} r_{i-1} & & \text { for } i \in\left[1, N^{\prime}-1\right] \cap I_{1}, \\
r_{0} h_{N-\frac{1}{2}}^{+} & =h_{N-\frac{1}{2}}^{+} h_{N-\frac{3}{2}}^{+} h_{N-\frac{3}{2}}^{-}, & & \\
h_{N^{\prime}+\frac{1}{2}}^{-} h_{N^{\prime}+\frac{1}{2}}^{+} h_{N^{\prime}-\frac{1}{2}}^{+} & =h_{N^{\prime}-\frac{1}{2}}^{+} r_{N^{\prime}-1}, & & \\
h_{i+\frac{3}{2}}^{-} h_{i+\frac{3}{2}}^{+} h_{i+\frac{1}{2}}^{+} & =h_{i+\frac{1}{2}}^{+} h_{i-\frac{1}{2}}^{+} h_{i-\frac{1}{2}}^{-} & & \text {for } i=\left[N^{\prime}+1, N-3\right] \cap I_{3} .
\end{aligned}
$$

As in Lemma A.8, once the value of $L$ is determined for $v_{l}^{a, b}(k)$ it is uniquely determined for all $H^{r} v_{l}^{a, b}(k)$ by the condition that the above relations be satisfied for the pair $\left(L, H\left(\pi^{[a, b]}\right)\right.$. To be precise, if $a \in I_{1}$ we have

and if $a \in I_{3}$ then

$$
L: H^{r}\left(v_{l}^{a, b}(k)\right) \mapsto \begin{cases}H^{r} L\left(v_{l}^{a, b}(k)\right) & \text { if } a+r \in I_{1}, \\ 0 & \text { if } a+r \in I_{2}, \\ H^{r-1} L\left(v_{l}^{a, b}(k)\right) & \text { if } a+r \in I_{3},\end{cases}
$$

$$
L: H^{r}\left(v_{l}^{a, b}(k)\right) \mapsto \begin{cases}H^{r+1} L\left(v_{l}^{a, b}(k)\right) & \text { if } a+r \in I_{1}, \\ 0 & \text { if } a+r \in I_{2}, \\ H^{r} L\left(v_{l}^{a, b}(k)\right) & \text { if } a+r \in I_{3} .\end{cases}
$$

Since $L \in F\left(\pi^{[a, b]}, \pi^{[c, d]}\right)$ by definition, its image must lie in the space $V^{c, d}$; also, if $a \in I_{1}$ then $L: V_{a} \rightarrow V_{a}$ and if $a \in I_{3}$ then $L: V_{a} \rightarrow V_{a-1}$. The only further condition on the image of a vector $v_{l}^{a, b}(k)$ is that its image be killed by a high-enough power of $H$. It is given that $H^{N \cdot(l-1)+|b-a|+1} v_{l}^{a, b}(k)=0$, so then $H^{t}\left(L v_{l}^{a, b}(k)\right)=0$, where the exponent $t$ is read off from the defining relations on $L$ above. In the separate cases,

$$
L v_{l}^{a, b}(k) \in\left\{\begin{array}{cl}
V_{a} \cap V^{N \cdot(l-1)+|b-a|+1} \cap V^{c, d} & \text { if } a \in I_{1} \text { and } b \notin I_{2}, \\
V_{a} \cap V^{N \cdot(l-1)+|b-a|} \cap V^{c, d} & \text { if } a \in I_{1} \text { and } b \in I_{2}, \\
V_{a-1} \cap V^{N \cdot(l-1)+|b-a|+2} \cap V^{c, d} & \text { if } a \in I_{3} \text { and } b \notin I_{2}, \\
V_{a-1} \cap V^{N \cdot(l-1)+|b-a|+1} \cap V^{c, d} & \text { if } a \in I_{3} \text { and } b \in I_{2},
\end{array}\right.
$$

proving the result.

We have a result similar to Lemma A.10 when $a \in I_{2}$.

Lemma A.11. Suppose $a \in I_{2}$ and $L \in F\left(\pi^{[a, b]}, \pi^{[c, d]}\right)$; then the map $L$ is uniquely determined by its value on the vectors $H v_{l}^{a, b}(k)$. Moreover, the only restriction on the image of such a vector is that it lie in a linear subspace:

$$
L\left(H v_{l}^{a, b}(k)\right) \in \begin{cases}V_{a} \cap V^{N \cdot(l-1)+|b-a|+1} \cap V^{c, d} & \text { if } b \notin I_{2}, \\ V_{a} \cap V^{N \cdot(l-1)+|b-a|} \cap V^{c, d} & \text { if } b \in I_{2} .\end{cases}
$$


Proof. Again, we know that to define the linear map $L$ on the space $V^{a, b}$ it suffices to define its value at each of the basis vectors

$$
\left\{H^{r} v_{l}^{a, b}(k)|0 \leq r \leq N \cdot(l-1)+| b-a \mid, 1 \leq k \leq b_{l}^{a, b}\right\} .
$$

Since by definition if $a \in I_{2}$ then $L v_{l}^{a, b}(k)=0$, the map is already trivially determined on these vectors and their image does not suffice to determine the map in general. However if we consider the vectors $H v_{l}^{a, b}(k)$, then once the value of $L$ is determined for $H v_{l}^{a, b}(k)$ it is uniquely determined for all $H^{r} v_{l}^{a, b}(k)$ by the condition that the relations (see Lemma A.10) be satisfied by the pair $\left(L, H\left(\pi^{[a, b]}\right)\right.$. To be precise, if $a \in I_{2}$ we have

$$
L: H^{r}\left(v_{l}^{a, b}(k)\right) \mapsto \begin{cases}H^{r} L\left(H v_{l}^{a, b}(k)\right) & \text { if } a+r \in I_{1}, \\ 0 & \text { if } a+r \in I_{2}, \\ H^{r-1} L\left(H v_{l}^{a, b}(k)\right) & \text { if } a+r \in I_{3} .\end{cases}
$$

By definition, we know that the image of $L$ lies in $V^{c, d}$ and also that for $a \in I_{2}$ we have $L: V_{a+1} \rightarrow V_{a}$. As before the only remaining condition on the image of $v_{l}^{a, b}(k)$ is that it be killed by a high-enough power of $H$. From the definition of $L$ above we see that

$$
L\left(H v_{l}^{a, b}(k)\right) \in \begin{cases}V_{a} \cap V^{N \cdot(l-1)+|b-a|+1} \cap V^{c, d} & \text { if } b \notin I_{2}, \\ V_{a} \cap V^{N \cdot(l-1)+|b-a|} \cap V^{c, d} & \text { if } b \in I_{2},\end{cases}
$$

proving the result.

The following notation encapsulates the dimensions of all the vector spaces encountered in the last two lemmas.

Definition A.12. We define integers

$d_{a, b: c, d}(l)$

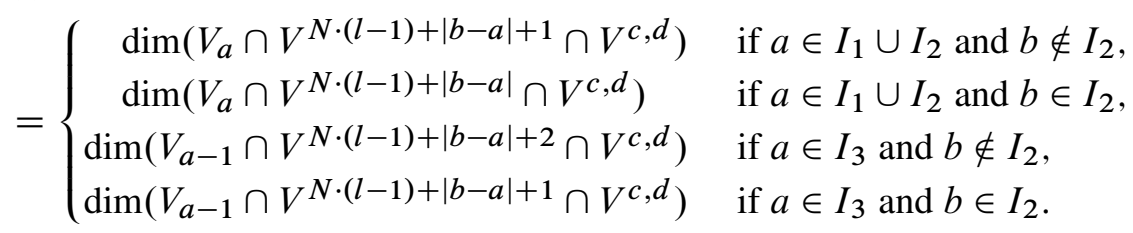

From Lemma A.10 and Lemma A.11 we deduce the dimension of the spaces $F\left(\pi^{[a, b]}, \pi^{[c, d]}\right)$.

Corollary A.13. If $a \in I_{1} \cup I_{2}$ and $b \notin I_{2}$ then

$T\left(\pi^{[a, b]}, \pi^{[c, d]}\right)= \begin{cases}M\left(\pi^{[a, b]}, \pi^{[c, d]}\right) & \text { if } a \in[c, d] \text { and }|d-a| \leq|b-a|, \\ M\left(\left(\pi^{[a, b]}\right)^{\prime}, \pi^{[c, d]}\right) & \text { if } a \in[c, d] \text { and }|d-a|>|b-a|, \\ M\left(\pi^{[a, b]},\left(\pi^{[c, d]}\right)^{\prime}\right) & \text { if } a \notin[c, d] \text { and }|d-a| \leq|b-a|, \\ M\left(\left(\pi^{[a, b]}\right)^{\prime},\left(\pi^{[c, d]}\right)^{\prime}\right) & \text { if } a \notin[c, d] \text { and }|d-a|>|b-a| .\end{cases}$ 
If $a \in I_{1} \cup I_{2}$ and $b \in I_{2}$ then

$$
T\left(\pi^{[a, b]}, \pi^{[c, d]}\right)= \begin{cases}M\left(\pi^{[a, b]}, \pi^{[c, d]}\right) & \text { if } a \in[c, d] \text { and }|d-a| \leq|b-a|-1, \\ M\left(\left(\pi^{[a, b]}\right)^{\prime}, \pi^{[c, d]}\right) & \text { if } a \in[c, d] \text { and }|d-a|>|b-a|-1, \\ M\left(\pi^{[a, b]},\left(\pi^{[c, d]}\right)^{\prime}\right) & \text { if } a \notin[c, d] \text { and }|d-a| \leq|b-a|-1, \\ M\left(\left(\pi^{[a, b]}\right)^{\prime},\left(\pi^{[c, d]}\right)^{\prime}\right) & \text { if } a \notin[c, d] \text { and }|d-a|>|b-a|-1 .\end{cases}
$$

If $a \in I_{3}$ and $b \notin I_{2}$ then

$$
\begin{aligned}
& T\left(\pi^{[a, b]}, \pi^{[c, d]}\right) \\
& \quad= \begin{cases}M\left(\pi^{[a, b]}, \pi^{[c, d]}\right) & \text { if } a-1 \in[c, d] \text { and }|d-(a-1)| \leq|b-a|+1, \\
M\left(\left(\pi^{[a, b]}\right)^{\prime}, \pi^{[c, d]}\right) & \text { if } a-1 \in[c, d] \text { and }|d-(a-1)|>|b-a|+1, \\
M\left(\pi^{[a, b]},\left(\pi^{[c, d]}\right)^{\prime}\right) & \text { if } a-1 \notin[c, d] \text { and }|d-(a-1)| \leq|b-a|+1, \\
M\left(\left(\pi^{[a, b]}\right)^{\prime},\left(\pi^{[c, d]}\right)^{\prime}\right) & \text { if } a-1 \notin[c, d] \text { and }|d-(a-1)|>|b-a|+1 .\end{cases}
\end{aligned}
$$

If $a \in I_{3}$ and $b \in I_{2}$ then

$$
\begin{aligned}
& T\left(\pi^{[a, b]}, \pi^{[c, d]}\right) \\
& = \begin{cases}M\left(\pi^{[a, b]}, \pi^{[c, d]}\right) & \text { if } a-1 \in[c, d] \text { and }|d-(a-1)| \leq|b-a|, \\
M\left(\left(\pi^{[a, b]}\right)^{\prime}, \pi^{[c, d]}\right) & \text { if } a-1 \in[c, d] \text { and }|d-(a-1)|>|b-a|, \\
M\left(\pi^{[a, b]},\left(\pi^{[c, d]}\right)^{\prime}\right) & \text { if } a-1 \notin[c, d] \text { and }|d-(a-1)| \leq|b-a|, \\
M\left(\left(\pi^{[a, b]}\right)^{\prime},\left(\pi^{[c, d]}\right)^{\prime}\right) & \text { if } a-1 \notin[c, d] \text { and }|d-(a-1)|>|b-a| .\end{cases}
\end{aligned}
$$

Proof. We know that if $a \in I_{1} \cup I_{3}$ (resp. $\left.a \in I_{2}\right)$ then the map $L \in F\left(\pi^{[a, b]}, \pi^{[c, d]}\right.$ ) is determined by its value at the vectors $v_{l}^{a, b}(k)\left(\right.$ resp. $\left.H v_{l}^{a, b}(k)\right)$ for $1 \leq k \leq b_{l}^{a, b}$. In the notation of the previous definition, such a vector takes values in a space of dimension $d_{a, b ; c, d}(l)$. So in all cases the total dimension of the space $F\left(\pi^{[a, b]}, \pi^{[c, d]}\right)$ is equal to

$$
T\left(\pi^{[a, b]}, \pi^{[c, d]}\right)=\sum_{l \geq 1} b_{l}^{a, b} \cdot d_{a, b ; c, d}(l)
$$

In the above definition of $d_{a, b ; c, d}(l)$ there are four possible forms, depending on the value of $a$ and $b$. Lets consider the first case, where $a \in I_{1} \cup I_{2}$ and $b \notin I_{2}$. Then we have that

$$
d_{a, b ; c, d}(l)=\operatorname{dim}\left(V_{a} \cap V^{N \cdot(l-1)+|b-a|+1} \cap V^{c, d}\right) .
$$


Counting the number of basis vectors of $V^{c, d}$ that lie in $V_{a}$, we see there are four possibilities for $\operatorname{dim}\left(V_{a} \cap V^{c, d} \cap V^{N \cdot(l-1)+|b-a|+1}\right)$ :

$$
\begin{array}{ll}
\sum_{i=1}^{l} i b_{i}^{c, d}+l \sum_{i \geq l} b_{i}^{c, d} & \text { if } a \in[c, d] \text { and }|d-a| \leq|b-a|, \\
\sum_{i=1}^{l-1} i b_{i}^{c, d}+(l-1) \sum_{i \geq l} b_{i}^{c, d} & \text { if } a \in[c, d] \text { and }|d-a|>|b-a|, \\
\sum_{i=1}^{l} i b_{i+1}^{c, d}+l \sum_{i \geq l} b_{i+1}^{c, d} & \text { if } a \notin[c, d] \text { and }|d-a| \leq|b-a|, \\
\sum_{i=1}^{l-1} i b_{i+1}^{c, d}+(l-1) \sum_{i \geq l} b_{i+1}^{c, d} & \text { if } a \notin[c, d] \text { and }|d-a|>|b-a| .
\end{array}
$$

In the first case $a \in[c, d]$ and $|d-a| \leq|b-a|$, we have

$$
\begin{aligned}
T\left(\pi^{[a, b]}, \pi^{[c, d]}\right) & =\sum_{l \geq 1} b_{l}^{a, b} \cdot\left(\sum_{i=1}^{l} i b_{i}^{c, d}+l \sum_{i \geq l} b_{i}^{c, d}\right) \\
& =\sum_{i \geq 1}\left(\sum_{l \geq i} b_{l}^{a, b}\right) \cdot\left(\sum_{l \geq i} b_{l}^{c, d}\right) \\
& =M\left(\pi^{[a, b]}, \pi^{[c, d]}\right) .
\end{aligned}
$$

In the second case $a \in[c, d]$ and $|d-a|>|b-a|$, we have

$$
\begin{aligned}
T\left(\pi^{[a, b]}, \pi^{[c, d]}\right) & =\sum_{l \geq 1} b_{l}^{a, b} \cdot\left(\sum_{i=1}^{l-1} i b_{i}^{c, d}+(l-1) \sum_{i \geq l} b_{i}^{c, d}\right) \\
& =\sum_{i \geq 1}\left(\sum_{l \geq i} b_{l+1}^{a, b}\right) \cdot\left(\sum_{l \geq i} b_{l}^{c, d}\right) \\
& =M\left(\left(\pi^{[a, b]}\right)^{\prime}, \pi^{[c, d]}\right) .
\end{aligned}
$$

In the third case $a \notin[c, d]$ and $|d-a| \leq|b-a|$, we have

$$
\begin{aligned}
T\left(\pi^{[a, b]}, \pi^{[c, d]}\right) & =\sum_{l \geq 1} b_{l}^{a, b} \cdot\left(\sum_{i=1}^{l} i b_{i+1}^{c, d}+l \sum_{i \geq l} b_{i+1}^{c, d}\right) \\
& =\sum_{i \geq 1}\left(\sum_{l \geq i} b_{l}^{a, b}\right) \cdot\left(\sum_{l \geq i} b_{l+1}^{c, d}\right) \\
& =M\left(\pi^{[a, b]},\left(\pi^{[c, d]}\right)^{\prime}\right) .
\end{aligned}
$$


Finally, in the fourth case $a \notin[c, d]$ and $|d-a|>|b-a|$, we have

$$
\begin{aligned}
T\left(\pi^{[a, b]}, \pi^{[c, d]}\right) & =\sum_{l \geq 1} b_{l}^{a, b} \cdot\left(\sum_{i=1}^{l-1} i b_{i+1}^{c, d}+(l-1) \sum_{i \geq l} b_{i+1}^{c, d}\right) \\
& =\sum_{i \geq 1}\left(\sum_{l \geq i} b_{l+1}^{a, b}\right) \cdot\left(\sum_{l \geq i} b_{l+1}^{c, d}\right) \\
& =M\left(\left(\pi^{[a, b]}\right)^{\prime},\left(\pi^{[c, d]}\right)^{\prime}\right) .
\end{aligned}
$$

This completes the situation when $a \in I_{1} \cup I_{2}$ and $b \notin I_{2}$. In the other situations, $a \in I_{1} \cup I_{2}$ and $b \in I_{2}$, or $a \in I_{3}$ and $b \notin I_{2}$, or $a \in I_{3}$ and $b \in I_{2}$. All of these cases can be dealt with in a similar manner.

Now we have computed all the dimensions $T\left(\pi^{[a, b]}, \pi^{[c, d]}\right)$ and $B\left(\pi^{[a, b]}, \pi^{[c, d]}\right)$. The next lemma combines Corollaries A.9 and A.13 to compute their difference. We see that in most cases there is an exact cancellation:

Lemma A.14. The equality

$$
T\left(\pi^{[a, b]}, \pi^{[c, d]}\right)=B\left(\pi^{[a, b]}, \pi^{[c, d]}\right)
$$

holds, except in the following cases, where we give the possible values of the difference $T\left(\pi^{[a, b]}, \pi^{[c, d]}\right)-B\left(\pi^{[a, b]}, \pi^{[c, d]}\right)$ :

Case 1: $a \in I_{1} \cup I_{2}, b=d \in I_{2}$ :

$$
\begin{array}{cc}
M\left(\left(\pi^{[a, b]}\right)^{\prime}, \pi^{[c, b]}\right)-M\left(\pi^{[a, b]}, \pi^{[c, b]}\right) & \text { if } a \in[c, b], \\
M\left(\left(\pi^{[a, b]}\right)^{\prime},\left(\pi^{[c, b]}\right)^{\prime}\right)-M\left(\pi^{[a, b]},\left(\pi^{[c, b]}\right)^{\prime}\right) & \text { if } a \notin[c, b] .
\end{array}
$$

Case 2: $a \in I_{3}, b \notin I_{2}, d=a-1 \in I_{2}$ :

$$
\begin{array}{ll}
M\left(\pi^{[a, b]}, \pi^{[a, a-1]}\right)-M\left(\left(\pi^{[a, b]}\right)^{\prime}, \pi^{[a, a-1]}\right) & \text { if } a=c, \\
M\left(\pi^{[a, b]}, \pi^{[c, a-1]}\right)-M\left(\left(\pi^{[a, b]}\right)^{\prime},\left(\pi^{[c, a-1]}\right)^{\prime}\right) & \text { if } a \neq c .
\end{array}
$$

Case 3: $a \in I_{3}, b \notin I_{2}, a=c, d \neq a-1$ :

$$
\begin{array}{cc}
M\left(\pi^{[a, b]},\left(\pi^{[a, d]}\right)^{\prime}\right)-M\left(\pi^{[a, b]}, \pi^{[a, d]}\right) & \text { if }|d-a| \leq|b-a|, \\
M\left(\left(\pi^{[a, b]}\right)^{\prime},\left(\pi^{[a, d]}\right)^{\prime}\right)-M\left(\left(\pi^{[a, b]}\right)^{\prime}, \pi^{[a, d]}\right) & \text { if }|d-a|>|b-a| .
\end{array}
$$

Case 4: $a \in I_{3}, b \in I_{2}, d=a-1$ :

$$
\begin{array}{cl}
M\left(\pi^{[a, b]}, \pi^{[a, a-1]}\right)-M\left(\left(\pi^{[a, b]}\right)^{\prime}, \pi^{[a, a-1]}\right) & \text { if } a=c \text { and } b \neq a-1, \\
M\left(\pi^{[a, a-1]}, \pi^{[c, a-1]}\right)-M\left(\pi^{[a, a-1]},\left(\pi^{[c, a-1]}\right)^{\prime}\right) & \text { if } a \neq c \text { and } b=a-1, \\
M\left(\pi^{[a, b]}, \pi^{[c, a-1]}\right)-M\left(\left(\pi^{[a, b]}\right)^{\prime},\left(\pi^{[c, a-1]}\right)^{\prime}\right) & \text { if } a \neq c \text { and } b \neq a-1 .
\end{array}
$$


Case 5: $a \in I_{3}, b \in I_{2}, a-1 \in[c, d], d \neq a-1, b=d$ :

$$
M\left(\left(\pi^{[a, b]}\right)^{\prime}, \pi^{[c, b]}\right)-M\left(\pi^{[a, b]}, \pi^{[c, b]}\right) .
$$

Case 6: $a \in I_{3}, b \in I_{2}, a-1 \notin[c, d], a=c,|d-a|<|b-a|$ :

$$
M\left(\pi^{[a, b]},\left(\pi^{[a, d]}\right)^{\prime}\right)-M\left(\pi^{[a, b]}, \pi^{[a, d]}\right) .
$$

Case 7: $a \in I_{3}, b \in I_{2}, a-1 \notin[c, d]$ :

$$
\begin{array}{ll}
M\left(\left(\pi^{[a, b]}\right)^{\prime},\left(\pi^{[a, b]}\right)^{\prime}\right)-M\left(\pi^{[a, b]}, \pi^{[a, b]}\right) & \text { if } a=c \text { and } b=d, \\
M\left(\left(\pi^{[a, b]}\right)^{\prime},\left(\pi^{[a, d]}\right)^{\prime}\right)-M\left(\left(\pi^{[a, b]}\right)^{\prime}, \pi^{[a, d]}\right) & \text { if } a=c \text { and }|d-a|>|b-a|, \\
M\left(\left(\pi^{[a, b]}\right)^{\prime},\left(\pi^{[c, b]}\right)^{\prime}\right)-M\left(\pi^{[a, b]},\left(\pi^{[c, b]}\right)^{\prime}\right) & \text { if } a \neq c \text { and } b=d .
\end{array}
$$

Proof. Compare Corollaries A.9 and A.13.

Our aim throughout this appendix has been to prove Proposition 4.18 and deduce that the difference $\sum_{0 \leq a, b, c, d \leq N-1} T\left(\pi^{[a, b]}, \pi^{[c, d]}\right)-B\left(\pi^{[a, b]}, \pi^{[c, d]}\right)$ is equal to

$$
\begin{aligned}
-\frac{1}{2} \sum_{i \in I_{2}}\left(\sum_{b \neq i} l\left(\pi^{[i+1, b]}\right)-\right. & \left.\sum_{c \neq i+1} l\left(\pi^{[c, i]}\right)\right)^{2} \\
& -\frac{1}{2} \sum_{a \in I_{3}, b \notin I_{2}} \sum_{i \geq 1}\left(b_{i}^{a, b}\right)^{2}-\frac{1}{2} \sum_{a \notin I_{3}, b \in I_{2}} \sum_{i \geq 1}\left(b_{i}^{a, b}\right)^{2} .
\end{aligned}
$$

So all that remains is to check this sum agrees with the values we computed. First we will transform it into a expression in terms of the $M\left(\pi^{[a, b]}, \pi^{[c, d]}\right)$. To do this we need the simple identities

$$
\begin{aligned}
M\left(\pi^{[a, b]}, \pi^{[c, d]}\right)-M\left(\left(\pi^{[a, b]}\right)^{\prime}\right. & \left.,\left(\pi^{[c, d]}\right)^{\prime}\right) \\
& =\sum_{l \geq 1}\left(\sum_{i \geq l} b_{i}^{a, b} \cdot \sum_{i \geq l} b_{i}^{c, d}-\sum_{i \geq l} b_{i+1}^{a, b} \cdot \sum_{i \geq l} b_{i+1}^{c, d}\right) \\
& =\sum_{i \geq 1} b_{i}^{a, b} \cdot \sum_{i \geq 1} b_{i}^{c, d} \\
& =l\left(\pi^{[a, b]}\right) \cdot l\left(\pi^{[c, d]}\right)
\end{aligned}
$$


and

$$
\begin{aligned}
M\left(\pi^{[a, b]}, \pi^{[a, b]}\right)-M\left(\left(\pi^{[a, b]}\right)^{\prime},\right. & \left.\pi^{[a, b]}\right) \\
& =\sum_{l \geq 1}\left(\sum_{i \geq l} b_{i}^{a, b} \cdot \sum_{i \geq l} b_{i}^{a, b}-\sum_{i \geq l} b_{i+1}^{a, b} \cdot \sum_{i \geq l} b_{i}^{a, b}\right) \\
& =\sum_{l \geq 1} b_{l}^{a, b} \cdot \sum_{i \geq l} b_{i}^{c, d} \\
& =\frac{1}{2} l\left(\pi^{[a, b]}\right)^{2}+\frac{1}{2} \sum_{l \geq 1}\left(b_{l}^{a, b}\right)^{2} .
\end{aligned}
$$

Using these two identities and some simple algebraic manipulations, we can rewrite Proposition 4.18 as the statement that the difference

$$
\sum_{0 \leq a, b, c, d \leq N-1} T\left(\pi^{[a, b]}, \pi^{[c, d]}\right)-B\left(\pi^{[a, b]}, \pi^{[c, d]}\right)
$$

is equal to

$$
\begin{aligned}
& \sum_{i \in I_{2}} \sum_{\substack{b \neq i \\
c \neq i+1}} M\left(\pi^{[i+1, b]}, \pi^{[c, i]}\right)-M\left(\left(\pi^{[i+1, b]}\right)^{\prime},\left(\pi^{[c, i]}\right)^{\prime}\right) \\
& +\sum_{i \in I_{2}} \sum_{\substack{b<d \\
b, d \neq i}} M\left(\left(\pi^{[i+1, b]}\right)^{\prime},\left(\pi^{[i+1, d]}\right)^{\prime}\right)-M\left(\pi^{[i+1, b]}, \pi^{[i+1, d]}\right) \\
& +\sum_{i \in I_{2}} \sum_{\substack{a<c \\
a, c \neq i+1}} M\left(\left(\pi^{[a, i]}\right)^{\prime},\left(\pi^{[c, i]}\right)^{\prime}\right)-M\left(\pi^{[a, i]}, \pi^{[c, i]}\right) \\
& +\sum_{\substack{a \in I_{3}, b \in I_{2} \\
b \neq a-1}} M\left(\left(\pi^{[a, b]}\right)^{\prime},\left(\pi^{[a, b]}\right)^{\prime}\right)-M\left(\pi^{[a, b]}, \pi^{[a, b]}\right) \\
& +\sum_{[a, b] \in S} M\left(\left(\pi^{[a, b]}\right)^{\prime}, \pi^{[a, b]}\right)-M\left(\pi^{[a, b]}, \pi^{[a, b]}\right) .
\end{aligned}
$$

By a careful systematic approach, one shows that all these terms are exactly accounted for in Lemma A.14.

\section{Acknowledgements}

Morrison thanks his PhD supervisor Jim Bryan for his mathematical support over the last years. Both authors thank Sergey Mozgovoy for valuable discussion. Morrison is partially supported by a Four Year Doctoral Fellowship (4YF), University of British Columbia. Nagao was supported by the Grant-in-Aid for Research Activity Start-up (no. 22840023) and for Scientific Research (S) (no. 22224001). 


\title{
Biographical note
}

\author{
by Prof. Hiraku Nakajima
}

Kentaro Nagao died on October 22, 2013, at age 31, from alveolar soft part sarcoma. He was a promising young mathematician, who started to make outstanding achievements in geometric representation theory. His too-early death was a sorrowful event and a big loss for mathematics.

Kentaro Nagao was born on April 18, 1982 in Tokyo, Japan. He first showed his mathematical ability by winning three gold medals $(1998,1999,2000)$ and one silver (1997) at the International Mathematical Olympiad. He studied at Kyoto University, where he specialized in representation theory and geometry, and received a PhD in mathematics in 2008 under H. Nakajima. He then spent half a year at the University of Oxford, working with D. Joyce. In Spring 2010 he was appointed to an assistant professorship at Nagoya University. He was awarded the MSJ Takebe Katahiro Prize for his works on Donaldson-Thomas theory and cluster algebras in September 2013, one month before his death. He did not lose his enthusiasm for research under his difficult medical condition, as he expressed in response to the award. But his research was tragically stopped. We miss him.

\section{References}

[Behrend 2009] K. Behrend, "Donaldson-Thomas type invariants via microlocal geometry", Ann. of Math. (2) 170:3 (2009), 1307-1338. MR 2011d:14098 Zbl 1191.14050

[Behrend and Fantechi 1997] K. Behrend and B. Fantechi, "The intrinsic normal cone", Invent. Math. 128:1 (1997), 45-88. MR 98e:14022 Zbl 0909.14006

[Behrend and Fantechi 2008] K. Behrend and B. Fantechi, "Symmetric obstruction theories and Hilbert schemes of points on threefolds", Algebra Number Theory 2:3 (2008), 313-345. MR 2009c: 14006 Zbl 1170.14004

[Behrend et al. 2013] K. Behrend, J. Bryan, and B. Szendrői, "Motivic degree zero DonaldsonThomas invariants”, Invent. Math. 192:1 (2013), 111-160. MR 3032328 Zbl 1267.14008

[Bryan and Morrison 2015] J. Bryan and A. Morrison, "Motivic classes of commuting varieties via power structures", J. Algebraic Geom. 24:1 (2015), 183-199. MR 3275657 Zbl 06387936

[Chevalley et al. 1958] C. Chevalley, A. Grothendieck, and J.-P. Serre, Séminaire C. Chevalley (2e année), III: Anneaux de Chow et applications, Secrétariat Mathématique, Paris, 1958. MR 22 \#1572 Zbl 0098.13101

[Denef and Loeser 2001] J. Denef and F. Loeser, "Geometry on arc spaces of algebraic varieties", pp. 327-348 in European Congress of Mathematics, I (Barcelona, 2000), edited by C. Casacuberta et al., Progr. Math. 201, Birkhäuser, Basel, 2001. MR 2004c:14037 Zbl 1079.14003

[Derksen et al. 2010] H. Derksen, J. Weyman, and A. Zelevinsky, "Quivers with potentials and their representations, II: Applications to cluster algebras", J. Amer. Math. Soc. 23:3 (2010), 749-790. MR 2012c:16044 Zbl 1208.16017

[Dimofte and Gukov 2010] T. Dimofte and S. Gukov, "Refined, motivic, and quantum", Lett. Math. Phys. 91:1 (2010), 1-27. MR 2011m:14094 Zbl 1180.81112 
[Getzler 1996] E. Getzler, "Mixed Hodge structures of configuration spaces", preprint, 1996. arXiv alg-geom/9510018

[Heinloth 2007] F. Heinloth, "A note on functional equations for zeta functions with values in Chow motives", Ann. Inst. Fourier (Grenoble) 57:6 (2007), 1927-1945. MR 2009b:14041 Zbl 1154.14018

[Iqbal et al. 2009] A. Iqbal, C. Kozçaz, and C. Vafa, "The refined topological vertex", J. High Energy Phys. 10 (2009), 069, 58. MR 2011d:81266

[Joyce and Song 2012] D. Joyce and Y. Song, A theory of generalized Donaldson-Thomas invariants, vol. 217, Mem. Amer. Math. Soc. 1020, Amer. Math. Soc., Providence, RI, 2012. MR 2951762 Zbl 1259.14054

[Keller and Yang 2011] B. Keller and D. Yang, "Derived equivalences from mutations of quivers with potential”, Adv. Math. 226:3 (2011), 2118-2168. MR 2012d:16043 Zbl 1272.13021

[Kontsevich and Soibelman 2008] M. Kontsevich and Y. Soibelman, "Stability structures, motivic Donaldson-Thomas invariants and cluster transformations", preprint, 2008. arXiv 0811:2435

[Looijenga 2002] E. Looijenga, "Motivic measures”, exposé no. 874, 267-297 in Séminaire Bourbaki 1999/2000 (Exposé 865-879), Astérisque 276, 2002. MR 2003k: 14010 Zbl 0996.14011

[Macdonald 1995] I. G. Macdonald, Symmetric functions and Hall polynomials, 2nd ed., Clarendon Press, Oxford University Press, New York, 1995. MR 96h:05207 Zbl 0824.05059

[Maulik et al. 2006] D. Maulik, N. Nekrasov, A. Okounkov, and R. Pandharipande, "Gromov-Witten theory and Donaldson-Thomas theory, I", Compos. Math. 142:5 (2006), 1263-1285. MR 2007i: 14061 Zbl 1108.14046

[Merkurjev 2013] A. S. Merkurjev, "Essential dimension: a survey", Transform. Groups 18:2 (2013), 415-481. MR 3055773 Zbl 1278.14066

[Morrison et al. 2012] A. Morrison, S. Mozgovoy, K. Nagao, and B. Szendrői, "Motivic DonaldsonThomas invariants of the conifold and the refined topological vertex", Adv. Math. 230:4-6 (2012), 2065-2093. MR 2927365 Zbl 1257.14028

[Nagao 2011a] K. Nagao, "Non-commutative Donaldson-Thomas theory and vertex operators", Geom. Topol. 15:3 (2011), 1509-1543. MR 2012i:14069 Zbl 1219.14066

[Nagao 2011b] K. Nagao, "Refined open noncommutative Donaldson-Thomas invariants for small crepant resolutions”, Pacific J. Math. 254:1 (2011), 173-209. MR 2900667 Zbl 1245.14053

[Nagao 2011c] K. Nagao, "Wall-crossing of the motivic Donaldson-Thomas invariants", preprint, 2011. arXiv 1103.2922

[Nagao 2012] K. Nagao, "Derived categories of small toric Calabi-Yau 3-folds and curve counting invariants”, Q. J. Math. 63:4 (2012), 965-1007. MR 2999994 Zbl 1259.14017

[Nagao 2013] K. Nagao, "Donaldson-Thomas theory and cluster algebras", Duke Math. J. 162:7 (2013), 1313-1367. MR 3079250 Zbl 06180315

[Nagao and Nakajima 2011] K. Nagao and H. Nakajima, "Counting invariant of perverse coherent sheaves and its wall-crossing”, Int. Math. Res. Not. 2011:17 (2011), 3885-3938. MR 2012h:14141 Zbl 1250.14021

[Okounkov et al. 2006] A. Okounkov, N. Reshetikhin, and C. Vafa, "Quantum Calabi-Yau and classical crystals", pp. 597-618 in The unity of mathematics, edited by P. Etingof et al., Progr. Math. 244, Birkhäuser, Boston, 2006. MR 2006k:81342 Zbl 1129.81080

[Pandharipande and Thomas 2009] R. Pandharipande and R. P. Thomas, "Curve counting via stable pairs in the derived category”, Invent. Math. 178:2 (2009), 407-447. MR 2010h:14089 Zbl 1204.14026 
[Segal 2008] E. Segal, "The $A_{\infty}$ deformation theory of a point and the derived categories of local Calabi-Yaus”, J. Algebra 320:8 (2008), 3232-3268. MR 2009k:16016 Zbl 1168.18005

[Szendrői 2008] B. Szendrôi, "Non-commutative Donaldson-Thomas invariants and the conifold", Geom. Topol. 12:2 (2008), 1171-1202. MR 2009e:14100 Zbl 1143.14034

[Thomas 2000] R. P. Thomas, "A holomorphic Casson invariant for Calabi-Yau 3-folds, and bundles on K3 fibrations", J. Differential Geom. 54:2 (2000), 367-438. MR 2002b:14049 Zbl 1034.14015

Communicated by Michel Van den Bergh

Received 2011-11-05 Revised 2012-04-26 Accepted 2015-03-27

andrewmo@math.ethz.ch ETH Zurich, $\mathrm{CH}$-8092 Zurich, Switzerland

Kentaro Nagao Graduate School of Mathematics, Nagoya University,

Furu-cho, Chikusa-Ku, Nagoya 464-8602, Japan 


\section{Algebra \& Number Theory}

msp.org/ant

\section{EDITORS}

MANAGING EDITOR

Bjorn Poonen

Massachusetts Institute of Technology

Cambridge, USA

\author{
EDITORIAL BOARD CHAIR \\ David Eisenbud \\ University of California \\ Berkeley, USA
}

\section{BOARD OF EDITORS}

Georgia Benkart

Dave Benson

Richard E. Borcherds

John H. Coates

J-L. Colliot-Thélène

Brian D. Conrad

Hélène Esnault

Hubert Flenner

Edward Frenkel

Andrew Granville

Joseph Gubeladze

Roger Heath-Brown

Craig Huneke

Kiran S. Kedlaya

János Kollár

Yuri Manin

Barry Mazur

Philippe Michel
University of Wisconsin, Madison, USA

University of Aberdeen, Scotland

University of California, Berkeley, USA

University of Cambridge, UK

CNRS, Université Paris-Sud, France

Stanford University, USA

Freie Universität Berlin, Germany

Ruhr-Universität, Germany

University of California, Berkeley, USA

Université de Montréal, Canada

San Francisco State University, USA

Oxford University, UK

University of Virginia, USA

Univ. of California, San Diego, USA

Princeton University, USA

Northwestern University, USA

Harvard University, USA

École Polytechnique Fédérale de Lausanne
Susan Montgomery

Shigefumi Mori

Raman Parimala

Jonathan Pila

Anand Pillay

Victor Reiner

Peter Sarnak

Joseph H. Silverman

Michael Singer

Vasudevan Srinivas

J. Toby Stafford

Ravi Vakil

Michel van den Bergh

Marie-France Vignéras

Kei-Ichi Watanabe

Efim Zelmanov

Shou-Wu Zhang
University of Southern California, USA

RIMS, Kyoto University, Japan

Emory University, USA

University of Oxford, UK

University of Notre Dame, USA

University of Minnesota, USA

Princeton University, USA

Brown University, USA

North Carolina State University, USA

Tata Inst. of Fund. Research, India

University of Michigan, USA

Stanford University, USA

Hasselt University, Belgium

Université Paris VII, France

Nihon University, Japan

University of California, San Diego, USA

Princeton University, USA

\section{PRODUCTION}

production@msp.org

Silvio Levy, Scientific Editor

See inside back cover or msp.org/ant for submission instructions.

The subscription price for 2015 is US $\$ 255 /$ year for the electronic version, and $\$ 440 /$ year ( $+\$ 55$, if shipping outside the US) for print and electronic. Subscriptions, requests for back issues and changes of subscribers address should be sent to MSP.

Algebra \& Number Theory (ISSN 1944-7833 electronic, 1937-0652 printed) at Mathematical Sciences Publishers, 798 Evans Hall \#3840, c/o University of California, Berkeley, CA 94720-3840 is published continuously online. Periodical rate postage paid at Berkeley, CA 94704, and additional mailing offices.

ANT peer review and production are managed by EditFLOW ${ }^{\circledR}$ from MSP.

\section{PUBLISHED BY}

- mathematical sciences publishers

nonprofit scientific publishing

http://msp.org/

(C) 2015 Mathematical Sciences Publishers 


\section{Algebra \& Number Theory}

Volume $9 \quad$ No. $4 \quad 2015$

Motivic Donaldson-Thomas invariants of small crepant resolutions

ANDREW MORRISON and KENTARO NAGAO

Étale homotopy equivalence of rational points on algebraic varieties

AMBRUS PÁL

Fermat's last theorem over some small real quadratic fields

NUNO FREITAS and SAMIR SIKSEK

Bounded negativity of self-intersection numbers of Shimura curves in Shimura surfaces MARTIN MÖLLER and DOMINGo TOLEDO

Singularities of locally acyclic cluster algebras

Angélica Benito, Greg Muller, Jenna Rajchgot and Karen E. Smith

On an analytic version of Lazard's isomorphism

GEORG TAMME

Towards local-global compatibility for Hilbert modular forms of low weight JAMES NEWTON

Horrocks correspondence on arithmetically Cohen-Macaulay varieties

981

Francesco Malaspina and A. Prabhakar RaO

The Elliott-Halberstam conjecture implies the Vinogradov least quadratic nonresidue conjecture

TERENCE TAO 